\title{
BERNSTEIN FUNCTIONS AND RATES IN MEAN ERGODIC THEOREMS FOR OPERATOR SEMIGROUPS
}

\author{
ALEXANDER GOMILKO, MARKUS HAASE, AND YURI TOMILOV
}

To Michael Lin on the occasion of his retirement

\begin{abstract}
We present a functional calculus approach to the study of rates of decay in mean ergodic theorems for bounded strongly continuous operator semigroups. A central role is played by operators of the form $g(A)$, where $-A$ is the generator of the semigroup and $g$ is a Bernstein function. In addition, we obtain some new results on Bernstein functions that are of independent interest.
\end{abstract}

\section{INTRODUCTION}

The famous mean ergodic theorem of von Neumann, Riesz, Kakutani, Lorch and Eberlein states that for a power-bounded operator $T$ on a reflexive Banach space $X$ the Cesàro averages

$$
\mathrm{A}_{n}(T)=\frac{1}{n} \sum_{j=0}^{n-1} T^{j}
$$

converge strongly as $n \rightarrow \infty$ to a bounded projection $P$ along $\overline{\operatorname{ran}}(I-T)$ onto the space $\operatorname{fix}(T)=\{x \in X \mid T x=x\}$ of fixed points. By works of Butzer and Westphal [5] and Browder [3], for $0 \neq x \in X$ the rate of convergence of $\mathrm{A}_{n}(T) x \rightarrow P x$ cannot be "better" than $\mathrm{O}(1 / n)$, and this optimal rate happens if and only if $x \in \operatorname{ran}(I-T)$. Moreover, by results of Dunford [18] and Lin [34, in the case that $\operatorname{ran}(I-T)$ is not closed there is no uniform rate of convergence working for all $x \in X$ simultaneously. However, one can ask for conditions on individual vectors $x$ to guarantee a certain rate, and Kachurovskii established in 29] - in the case of a unitary operator $T$ on a Hilbert space - connections between certain decay rates of $\mathrm{A}_{n}(T) x$ and the spectral measure of $x$ with respect to $T$.

In 2001, Derriennic and Lin - motivated by applications to central limit theorems for Markov chains and the quest for rates in the strong law of large numbers - opened a new chapter by addressing the problem of relating a prescribed decay rate for $\mathrm{A}_{n}(T) x$ with the convergence at $x$ of a certain power series in $T$. The case of polynomial rates could be settled already in [17] but some pertinent problems remained open, leading to a series of subsequent papers [1, [9]-16]. In particular, it was asked in [1] whether the (weak) convergence of the power series $\sum_{n=1}^{\infty} T^{n} x / n$ (the so-called one-sided ergodic Hilbert transform of $x$ ) would imply that $\left\|\mathrm{A}_{n}(T) x\right\|=\mathrm{O}(1 / \log n)$. (A spectral characterization for this rate when $T$ is unitary was given in [20] and [1], the case of normal $T$ was settled in [11.) Taking

Date: November 1, 2018.

1991 Mathematics Subject Classification. Primary 47A60, 47A35; Secondary 47D03.

Key words and phrases. mean ergodic theorem, rate of convergence, functional calculus, $C_{0^{-}}$ semigroup. 
into account the main results from [7] and 24], the question can be reformulated as whether the Cesàro means $\mathrm{A}_{n}(T) x$ decay logarithmically if $x \in \operatorname{dom}(\log (I-T))$. This question was recently answered positively by the authors in 22. Moreover, based on ideas from functional calculus theory, a general method was given in [22] to identify subspaces where certain rates hold.

In the present paper the analogous problems for bounded $C_{0}$-semigroups are discussed. Although the topic is very natural, to the best of our knowledge the only paper in this direction so far is [30]. However, as in the earlier work [29] it is confined to unitary operators and spectral methods, so its thrust is quite different from ours. Related results, but in the framework of real interpolation spaces can be found in [42, 39], and [4].

To set the stage, let $-A$ be the generator of a bounded $C_{0}$-semigroup $T:=$ $(T(s))_{s \geq 0}$ on a complex Banach space $X$. We shall study the asymptotic behaviour of the Cesàro averages

$$
\mathrm{C}_{t}(A) x:=\frac{1}{t} \int_{0}^{t} T(s) x \mathrm{~d} s \quad(x \in X),
$$

as $t \rightarrow \infty$. It is easy to show that for $x, y \in X$

$$
\mathrm{C}_{t}(A) x \rightarrow 0 \quad \Longleftrightarrow \quad x \in \overline{\operatorname{ran}}(A)
$$

and

$$
\mathrm{C}_{t}(A) x \rightarrow y \quad \Longrightarrow \quad y \in \operatorname{ker}(A)
$$

Therefore, $\operatorname{ker}(A) \oplus \overline{\operatorname{ran}}(A)$ is precisely the subspace of $X$ on which the Cesàro averages converge strongly, and the semigroup $(T(s))_{s \geq 0}$ is called mean ergodic if $X=\operatorname{ker}(A) \oplus \overline{\operatorname{ran}}(A)$ or, equivalently, $\mathrm{C}_{t}(A)$ converge strongly on $X$, see [25, Theorem 18.7.3] or [19, Section V.4]. A mean ergodic theorem provides conditions for a semigroup to be mean ergodic; for instance, a classical result states that every bounded $C_{0}$-semigroup on a reflexive space is mean ergodic [19, Example V.4.7].

In this paper we shall study rates of convergence for $\mathrm{C}_{t}(A) x$ as $t \rightarrow \infty$. Note that

$$
\operatorname{ker}(A)=\operatorname{fix}(T):=\{x \in X \mid T(s) x=x \forall s>0\} .
$$

If $\mathrm{C}_{t}(A) x \rightarrow y$ then $y \in \operatorname{ker}(A)$ and hence $\mathrm{C}_{t}(A) x-y=\mathrm{C}_{t}(A)(x-y)$. Thus, in the study of rates of the convergence of Cesàro averages one may confine oneself to the convergence to zero on $\overline{\operatorname{ran}}(A)$. By restricting $A$ to this $T$-invariant subspace, there is no loss of generality in assuming that $X=\overline{\operatorname{ran}}(A)$.

Let us recall now some known facts.

Proposition 1.1. Let $-A$ be the generator of a bounded $C_{0}$-semigroup $(T(s))_{s \geq 0}$ on a Banach space $X$. Then the following statements hold.

a) If $\left\|\mathrm{C}_{t}(A) x\right\|=\mathrm{o}(1 / t)$ as $t \rightarrow \infty$, then $x=0$.

b) If $x \in \operatorname{ran}(A)$ then $\left\|\mathrm{C}_{t}(A) x\right\|=\mathrm{O}(1 / t)$ as $t \rightarrow \infty$, and the converse is true if $X$ is reflexive.

c) If there exists a positive function $\varphi:(0, \infty) \rightarrow(0, \infty)$ such that $\varphi(t) \searrow 0$ as $t \rightarrow \infty$, and $\left\|\mathrm{C}_{t}(A) x\right\|=\mathrm{O}(\varphi(t)), t \rightarrow \infty$, for every $x \in X$, then $A$ is invertible. 
Part a) is due, essentially, to Butzer and Westphal [5. Actually, it follows easily from the formula

$$
A(I+A)^{-1} \frac{1}{t} \int_{0}^{t} s \mathrm{C}_{s}(A) x \mathrm{~d} s=(I+A)^{-1} x-(I+A)^{-1} \mathrm{C}_{t}(A) x \quad(t>0)
$$

and the boundedness of the operator $A(I+A)^{-1}$. This result tells us that we cannot have better convergence rates than $\mathrm{O}(1 / t)$. The first assertion in part $\mathrm{b}$ ) is straightforward, and the second one is obtained in [33. Theorem 2.8], see also [21. For the proof of c) one first concludes that $\lim _{t \rightarrow \infty} \mathrm{C}_{t}(A)=0$ in operator norm by the principle of uniform boundedness. Hence $T$ is a so-called uniformly ergodic semigroup, and Lin has proved in [35] that for such operators $\operatorname{ran}(A)$ must be closed. At the same time, under the assumption in $\mathrm{c}$ ), we have $\operatorname{ker}(A)=\{0\}$ and $\overline{\operatorname{ran}}(A)=X$.

Actually, modifying Lin's arguments one can sharpen this result to show that $A$-smoothness of a vector $x \in X$ has no influence on the asymptotics of $\mathrm{C}_{t}(A) x$, see Theorem A.1 below.

The theory in the discrete case as developed in 22 hinges on the notion of an admissible function, and one of the major difficulties was to find a continuous analogue for it. As it turned out, the well-studied notion of a Bernstein function provides such an analogue. However, the continuous theory is by no means a straightforward translation of the discrete theory, due to the fact that the generator $-A$ of a $C_{0}$-semigroup is usually unbounded. More severely and very much opposed to the discrete case, the operators $g(A)$, where $g$ is a Bernstein function, are usually unbounded as well. Dealing with this problem required a more sophisticated use of functional calculus theory and some new results about Bernstein functions, probably of independent interest.

As a result, we can cover polynomial and logarithmic rates. Employing the notion of a special Bernstein function and using ideas from [8], the lower estimates for rates could be improved with respect to 22 to the extent that they now apply under the sole condition that 0 is an accumulation point of the spectrum of the generator. Moreover, in addition to what was considered in [22, in this paper we characterize the functions that arise as the rates of decay for Cesàro means in our setting (Theorem 3.2).

Here is a synopsis of our main results: Given a Bernstein function $g$ we establish a uniform rate $r(t)$ of decay of $\mathrm{C}_{t}(A) x$ for $x \in \operatorname{ran}(g(A))$ (Theorem 3.4). Then we characterize those rate functions $r$ that are associated with a Bernstein function in this manner (Corollary 3.5 and Appendix B). Next, we show that the rate $r$ associated with a Bernstein function $g$ can be read off from $g$ without recurring on $g$ 's representing measure, which is unknown in most cases (Proposition 4.2). Then we prove that the (weak Abel) convergence of a certain integral of the orbit $(T(s) x)_{s \geq 0}$ implies that $x \in \operatorname{ran}(g(A))$, and hence that $\mathrm{C}_{t}(A) x$ has rate $r(t)$ (Theorem 4.4). Finally we show that our results are sharp under natural spectral assumptions, see Theorem 4.6 and Remark 4.8. In Section 5 we illustrate our approach with examples for polynomial and logarithmic rates.

1.1. Some Notations and Definitions. For a closed linear operator $A$ on a complex Banach space $X$ we denote by $\operatorname{dom}(A), \operatorname{ran}(A), \operatorname{ker}(A)$, and $\sigma(A)$ the domain, the range, the kernel, and the spectrum of $A$, respectively. The normclosure of the range is written as $\overline{\operatorname{ran}}(A)$. The space of bounded linear operators on 
$X$ is denoted by $\mathcal{L}(X)$. Let $\mathbb{R}_{+}$stand for $[0, \infty)$, and let $\mathrm{M}\left(\mathbb{R}_{+}\right)$denote the space of bounded Radon measures on $\mathbb{R}_{+}$. We write $\mathbb{C}_{+}:=\{z \in \mathbb{C} \mid \operatorname{Re} z>0\}$ for the open and $\overline{\mathbb{C}}_{+}:=\{z \in \mathbb{C} \mid \operatorname{Re} z \geq 0\}$ for the closed right halfplane. For positive functions $r(t), t \geq 0$, and $s(t), t \geq 0$, we write $r \sim s$ if there is $c>0$ such that $r(t) / c \leq s(t) \leq c r(t)$ for sufficiently large $t \in \mathbb{R}_{+}$.

\section{Preliminaries}

2.1. Laplace Transforms. A complex Radon measure $\mu$ on $\mathbb{R}_{+}$is called Laplace transformable if

$$
\int_{\mathbb{R}_{+}} e^{-s t}|\mu|(\mathrm{d} s)<\infty \quad \text { for each } t>0 .
$$

Let us write $\mathrm{e}_{z}(s):=e^{-s z}$ for $z \in \mathbb{C}$ and $s \geq 0$. Then $\mu$ is Laplace-transformable if $\mathrm{e}_{t} \mu \in \mathrm{M}\left(\mathbb{R}_{+}\right)$for each $t>0$. The Laplace-transformable complex Radon measures form a Fréchet space. The Laplace transform of a Laplace-transformable complex Radon measure $\mu$ on $\mathbb{R}_{+}$is

$$
(\mathcal{L} \mu)(z)=\widehat{\mu}(z):=\int_{\mathbb{R}_{+}} e^{-s z} \mu(\mathrm{d} s) \quad(\operatorname{Re} z>0) .
$$

If $\mu$ is a bounded measure, then $\mathcal{L} \mu$ has an extension to a continuous function on $\overline{\mathbb{C}}_{+}$. The space

$$
\mathrm{A}_{+}^{1}\left(\mathbb{C}_{+}\right):=\left\{\mathcal{L} \mu \mid \mu \in \mathrm{M}\left(\mathbb{R}_{+}\right)\right\}
$$

is a Banach algebra with respect to the pointwise multiplication norm

$$
\|\mathcal{L} \mu\|_{\mathrm{A}_{+}^{1}}:=\|\mu\|_{\mathrm{M}\left(\mathbb{R}_{+}\right)}=|\mu|\left(\mathbb{R}_{+}\right)
$$

and the Laplace transform

$$
\mathcal{L}: \mathrm{M}\left(\mathbb{R}_{+}\right) \longrightarrow \mathrm{A}_{+}^{1}\left(\mathbb{C}_{+}\right)
$$

is an isometric isomorphism. Indeed, $\mathrm{M}\left(\mathbb{R}_{+}\right)$is a (unital) Banach algebra with multiplication given by convolution and with the norm defined by (2.1) (see [25, p. 141-144]), and the Laplace transform is an injective algebra homomorphism from $\mathrm{M}\left(\mathbb{R}_{+}\right)$to $\mathrm{A}_{+}^{1}\left(\mathbb{C}_{+}\right)$.

More general, if $\mu, \nu$ are Laplace-transformable, then their convolution $\mu * \nu$ exists and is again Laplace-transformable. This follows from the identity

$$
\mathrm{e}_{t}(\mu * \nu)=\left(\mathrm{e}_{t} \mu\right) *\left(\mathrm{e}_{t} \nu\right)
$$

which is true for bounded measures, and can serve as a basis for defining $\mu * \nu$ if $\mu$ or $\nu$ is not a bounded measure. A simple computation then yields the identity

$$
\mathcal{L}(\mu * \nu)=(\mathcal{L} \mu) \cdot(\mathcal{L} \nu)
$$

for all Laplace-transformable Radon measures $\mu, \nu$ on $\mathbb{R}_{+}$.

2.2. Functional Calculus. Let $-A$ be the generator of a bounded $C_{0}$-semigroup $(T(s))_{s \geq 0}$ on a Banach space $X$. Recall that at least heuristically ' $T(s)=e^{-s A}$ '. Keeping this in mind, observe that the assignment

$$
g=\widehat{\mu}=\int_{\mathbb{R}_{+}} e^{-s z} \mu(\mathrm{d} s) \mapsto g(A):=\int_{\mathbb{R}_{+}} T(s) \mu(\mathrm{d} s)
$$


(with a strong integral in the definition of $g(A)$ ) is a continuous algebra homomorphism of $\mathrm{A}_{+}^{1}\left(\mathbb{C}_{+}\right)$into $\mathcal{L}(X)$ satisfying

$$
\left.\|g(A)\| \leq \sup _{s \geq 0}\|T(s)\|\right)\|g\|_{\mathrm{A}_{+}^{1}} \quad\left(g \in \mathrm{A}_{+}^{1}\left(\mathbb{C}_{+}\right)\right) .
$$

This homomorphism is called the Hille-Phillips (HP) functional calculus for A, see [25. Chapter $\mathrm{XV}]$. It has a canonical extension towards a larger function class, yielding unbounded operators in general. This extension is constructed via the so-called regularization method as follows: if $f: \mathbb{C}_{+} \rightarrow \mathbb{C}$ is holomorphic such that there exists a function $e \in \mathrm{A}_{+}^{1}\left(\mathbb{C}_{+}\right)$with ef $\in \mathrm{A}_{+}^{1}\left(\mathbb{C}_{+}\right)$and the operator $e(A)$ is injective, then

$$
f(A):=e(A)^{-1}(e f)(A)
$$

with its natural domain $\operatorname{dom}(f(A)):=\{x \in X \mid(e f)(A) x \in \operatorname{ran}(e(A))\}$. In this case $f$ is called regularizable, and $e$ is called a regularizer for $f$. It is easily shown that the definition of $f(A)$ does not depend on the chosen regularizer $e$ and that $f(A)$ is a closed (but possibly unbounded) operator on $X$. Moreover, the set of all regularizable functions $f$ is an algebra (depending on $A$ ). (See e.g. [23, p. 4-5] and [15. p. 246-249].) The assignment

$$
f \longmapsto f(A)
$$

from this algebra into the set of all closed operators on $X$ is called the extended Hille-Phillips calculus for $A$. There are natural rules governing this calculus, see for example [23, Chapter 1], the most important of which is the product rule: if $f$ is regularizable and $g \in \mathrm{A}_{+}^{1}\left(\mathbb{C}_{+}\right)$, then

$$
g(A) f(A) \subseteq f(A) g(A)=(f g)(A),
$$

where we take the natural domain for a product of operators, and inclusion means inclusion of graphs, i.e., extension. In particular, it follows that $(f g)(A) \in \mathcal{L}(X)$ if and only if $\operatorname{ran}(g(A)) \subseteq \operatorname{dom}(f(A))$.

While the explicit description of the domain of $f(A)$ could be rather nontrivial, one can recover $f(A)$ from its restriction to $\operatorname{dom}\left(A^{n}\right), n \in \mathbb{N}$, as the following lemma shows.

Lemma 2.1. Let $-A$ be the generator of a bounded $C_{0}$-semigroup on a Banach space $X$, and let $f(A)$ be defined in the extended HP-calculus for $A$. Then for each $n \in \mathbb{N}$ the space

$$
\mathcal{I}:=\left\{x \in \operatorname{dom}\left(A^{n}\right) \cap \operatorname{dom}(f(A)) \mid f(A) x \in \operatorname{dom}\left(A^{n}\right)\right\}
$$

is a core for $f(A)$, that is the closure of the restriction of $f(A)$ to $\mathcal{I}$ is $f(A)$ itself. Proof. Note that $r_{t}(z):=(t+z)^{-1} \in \mathrm{A}_{+}^{1}\left(\mathbb{C}_{+}\right)$for each $t>0$. By (2.3), if $x \in$ $\operatorname{dom}(f(A))$ and $f(A) x=y$ then $f(A)\left[t^{n}(t+A)^{-n}\right] x=t^{n}(t+A)^{-n} y$. This shows that $t^{n}(t+A)^{-n} x \in \operatorname{dom}(f(A)) \cap \operatorname{dom}\left(A^{n}\right)$, and since $\operatorname{dom}(A)$ is dense we have $t^{n}(t+A)^{-n} x \rightarrow x$ as $t \rightarrow \infty$, and the same holds for $y$. As the operator $f(A)$ is closed this completes the proof.

The following spectral inclusion theorem is well-known [25, Theorem 16.3.5]. For the convenience of the reader we provide a (particularly simple) proof.

Theorem 2.2. Let $g \in \mathrm{A}_{+}^{1}\left(\mathbb{C}_{+}\right)$and let $-A$ be the generator of a bounded $C_{0}$ semigroup $(T(s))_{s \geq 0}$ on a Banach space $X$. Then

$$
\{g(\lambda) \mid \lambda \in \sigma(A)\}=g(\sigma(A)) \subseteq \sigma(g(A)) .
$$


Proof. Let $\lambda \in \sigma(A)$ and $g=\mathcal{L} \mu$ for some $\mu \in \mathrm{M}\left(\mathbb{R}_{+}\right)$. Note that $\operatorname{Re} \lambda \geq 0$, since the semigroup is bounded. Hence we have

$$
g(\lambda)-g(z)=\lim _{N \rightarrow \infty} \int_{0}^{N}\left(e^{-s \lambda}-e^{-s z}\right) \mu(\mathrm{d} s)
$$

the convergence being in the norm of $\mathrm{A}_{+}^{1}\left(\mathbb{C}_{+}\right)$. Furthermore,

$$
\begin{aligned}
\int_{0}^{N}\left(e^{-s \lambda}-e^{-s z}\right) \mu(\mathrm{d} s) & =-(\lambda-z) \int_{0}^{N} e^{-s \lambda} \int_{0}^{s} e^{t(\lambda-z)} \mathrm{d} t \mu(\mathrm{d} s) \\
& =-(\lambda-z) \int_{0}^{N}\left(e^{t \lambda} \int_{t}^{N} e^{-s \lambda} \mu(\mathrm{d} s)\right) e^{-t z} \mathrm{~d} t \\
& =(\lambda-z) h_{N}(z)
\end{aligned}
$$

for some $h_{N} \in \mathrm{A}_{+}^{1}\left(\mathbb{C}_{+}\right)$. By (2.3)

$$
\left[(\lambda-z) h_{N}\right](A)=(\lambda-A) h_{N}(A)
$$

hence if $g(\lambda)-g(A)$ is invertible then for big enough $N>0$ the operator $(\lambda-$ A) $h_{N}(A)$ is invertible as well. Since $h_{N}(A)(\lambda-A) \subseteq(\lambda-A) h_{N}(A)$ by (2.3) again, we conclude that $\lambda-A$ is invertible.

Suppose that $f=\mathcal{L} \mu$ for some Laplace-transformable but not necessarily bounded measure $\mu$. It is then natural to examine the operator

$$
x \longmapsto \int_{0}^{\infty} T(s) x \mu(\mathrm{d} s)
$$

defined on the set of $x \in X$ where this integral exists in whatever generalized sense. The next theorem roughly states that in case of "weak Abel summability" of the integral, this operator is in coherence with the extended HP-calculus.

Theorem 2.3. Let $\mu$ be a Laplace-transformable complex Radon measure on $\mathbb{R}_{+}$, and let $f:=\mathcal{L} \mu$. Suppose that $e \in \mathrm{A}_{+}^{1}\left(\mathbb{C}_{+}\right)$is such that ef $\in \mathrm{A}_{+}^{1}\left(\mathbb{C}_{+}\right)$as well. Let $-A$ be the generator of a bounded $C_{0}$-semigroup $(T(s))_{s \geq 0}$ on a Banach space $X$, and let $x, y \in X$ be such that

$$
\lim _{\alpha \searrow 0} \int_{0}^{\infty} e^{-\alpha s} T(s) x \mu(\mathrm{d} s)=y \quad \text { weakly } .
$$

Then $(e f)(A) x=e(A) y$.

Proof. Since $\mu$ is Laplace transformable, $\mathrm{e}_{\alpha} \mu \in \mathrm{M}\left(\mathbb{R}_{+}\right)$for each $\alpha>0$, and hence

$$
\int_{0}^{\infty} e^{-\alpha s} T(s) \mu(\mathrm{d} s)=\mathcal{L}\left(\mathrm{e}_{\alpha} \mu\right)(A) .
$$

Let $\nu \in \mathrm{M}\left(\mathbb{R}_{+}\right)$such that $\mathcal{L} \nu=e$. Then, since ef $\in \mathrm{A}_{+}^{1}\left(\mathbb{C}_{+}\right), \nu * \mu \in \mathrm{M}\left(\mathbb{R}_{+}\right)$. Moreover,

$$
\mathrm{e}_{\alpha} \nu \rightarrow \nu \quad \text { and } \quad \mathrm{e}_{\alpha}(\nu * \mu) \rightarrow \nu * \mu \quad \text { as } \alpha \searrow 0
$$

in the norm of $\mathrm{M}\left(\mathbb{R}_{+}\right)$. Consequently, $\mathcal{L}\left(\mathrm{e}_{\alpha} \nu\right)(A) \rightarrow e(A)$ in operator norm and hence

weakly. On the other hand,

$$
\mathcal{L}\left(\mathrm{e}_{\alpha} \nu\right)(A) \mathcal{L}\left(\mathrm{e}_{\alpha} \mu\right)(A) x \rightarrow e(A) y
$$

$$
\mathcal{L}\left(\mathrm{e}_{\alpha} \nu\right) \mathcal{L}\left(\mathrm{e}_{\alpha} \mu\right)=\mathcal{L}\left(\left(\mathrm{e}_{\alpha} \nu\right) *\left(\mathrm{e}_{\alpha} \mu\right)\right)=\mathcal{L}\left(\mathrm{e}_{\alpha}(\nu * \mu)\right) \rightarrow \mathcal{L}(\nu * \mu)=e f
$$

in the norm of $\mathrm{A}_{+}^{1}\left(\mathbb{C}_{+}\right)$. Inserting $A$ concludes the proof. 
Corollary 2.4. Let $f=\mathcal{L} \mu$ and let $A,(T(s))_{s \geq 0}, x, y$ as in Theorem 2.3. Suppose that $g$ is a (regularizable) holomorphic function on $\mathbb{C}_{+}$so that $g(A)$ is defined by the extended HP-calculus and $g f \in \mathrm{A}_{+}^{1}\left(\mathbb{C}_{+}\right)$. Then $y \in \operatorname{dom}(g(A))$ and $(g f)(A) x=$ $g(A) y$.

Proof. Take any $e \in \mathrm{A}_{+}^{1}\left(\mathbb{C}_{+}\right)$such that $e g \in \mathrm{A}_{+}^{1}\left(\mathbb{C}_{+}\right)$. By Theorem 2.3 ,

$$
e(A)(g f)(A) x=(e g f)(A) x=(e g)(A) y .
$$

If $e$ is a regularizer for $g$, then $e(A)$ is injective, and we can conclude that $(g f)(A) x=$ $e(A)^{-1}(e g)(A) y$. Hence $y \in \operatorname{dom}(g(A))$ and $g(A) y=(g f)(A) x$.

We note that the weak Abel summability (2.4) is weaker than improper weak summability

$$
\lim _{r \rightarrow \infty} \int_{0}^{r} T(s) x \mu(\mathrm{d} s)=y \quad \text { weakly. }
$$

Indeed, this follows by applying elements from the dual space and employing the regularity of scalar Abel summability [43, p.181].

Actually, (2.4) is in general even strictly weaker than (2.5). As an example consider the case $f(z)=1 / z$, i.e., $\mu$ is ordinary Lebesgue measure. Then (2.4) just means that

$$
\lim _{\alpha \searrow 0} \int_{0}^{\infty} e^{-\alpha s} T(s) x \mathrm{~d} s=\lim _{\alpha \searrow 0}(\alpha+A)^{-1} x=y \quad \text { weakly. }
$$

Taking $g(z)=z$ in Corollary 2.4 we obtain $x=A y$; conversely, it is easily seen that $x \in \operatorname{ran}(A)$ implies (2.6), cf. [33, Theorem 2.1]. However, for $x=A z \in \operatorname{ran}(A)$ one has

$$
\int_{0}^{r} T(s) x \mu(\mathrm{d} s)=z-T(r) z,
$$

and hence (2.5) holds if and only if $\lim _{r \rightarrow \infty} T(r) z=y-z$ weakly. Hence every multiplication semigroup $T(s)=e^{i a s} I$ for $a \in \mathbb{R} \backslash\{0\}$ is an example for when (2.4) and (2.5) differ. (See Remark 4.5 below for more about this topic.)

Bernstein Functions. We now set up a functional-analytic background needed for our studies of rates. As a general reference for most of material in the next subsections we use the recent book [41.

The notions of completely monotone and Bernstein functions are essential for our approach. A function $f \in \mathrm{C}^{\infty}(0, \infty)$ is called completely monotone if

$$
f(t) \geq 0 \quad \text { and } \quad(-1)^{n} \frac{\mathrm{d}^{n} f(t)}{\mathrm{d} t^{n}} \geq 0 \quad \text { for all } n \in \mathbb{N} \text { and } t>0 .
$$

By Bernstein's theorem [41, Theorem 1.4], a function $f \in \mathrm{C}^{\infty}(0, \infty)$ is completely monotone if and only if there exists a (necessarily unique) Laplace-transformable positive Radon measure $\mu$ on $\mathbb{R}_{+}$such that $f(t)=(\mathcal{L} \mu)(t)$ for all $t>0$.

A function $g \in \mathrm{C}^{\infty}(0, \infty)$ is called a Bernstein function if

$$
g(t) \geq 0 \quad \text { and } \quad(-1)^{n} \frac{\mathrm{d}^{n} g(t)}{\mathrm{d} t^{n}} \leq 0 \quad \text { for all } n \in \mathbb{N} \text { and } t>0 .
$$

By [41, Theorem 3.2], a function $g$ is a Bernstein function if and only if there exist constants $a, b \geq 0$ and a positive Radon measure $\mu$ on $(0, \infty)$ satisfying

$$
\int_{0+}^{\infty} \frac{s}{1+s} \mu(\mathrm{d} s)<\infty
$$


and such that

$$
g(z)=a+b z+\int_{0+}^{\infty}\left(1-e^{-s z}\right) \mu(\mathrm{d} s) \quad(z>0) .
$$

The triple $(a, b, \mu)$ is uniquely determined by the corresponding Bernstein function $g$. Note that from the definition of $g$ it follows that $g$ extends analytically to $\mathbb{C}_{+}$ and, moreover, $g \in \mathrm{C}\left(\overline{\mathbb{C}}_{+}\right)$(see [41, Proposition 3.5] and cf. Lemma 2.5 below). Since such an analytic extension is unique by standard complex function theory, there is no harm in identifying Bernstein functions with their extensions to $\mathbb{C}_{+}$, and we shall henceforth do so.

Clearly, a Bernstein function $g \sim(a, b, \mu)$ is positive, increasing, and satisfies

$$
a=g(0+) \quad \text { and } \quad b=\lim _{t \rightarrow \infty} \frac{g(t)}{t} .
$$

The Bernstein function $g$ is bounded if and only if $b=0$ and $\mu(0, \infty)<\infty$ 41, Corollary 3.7]. If $f$ is completely monotone and $g$ is a Bernstein function, then $f \circ g$ is completely monotone [41, Theorem 3.6]. In particular, if $0 \neq g$ is a Bernstein function then

$$
\frac{1}{g}=\frac{1}{z} \circ g
$$

is completely monotone, hence by Bernstein's theorem there is a positive Laplace transformable Radon measure $\nu$ with $1 / g=\mathcal{L} \nu$. A completely monotone function $f$ is called a potential if it is of the form $f=1 / g$ for some Bernstein function $g \neq 0$ [41, Definition 5.17]. (An analogous notion was introduced and studied in [6] where the representing measure was called completely positive.) If $f$ is a potential, then $f$ is decreasing with

$$
\lim _{t \rightarrow \infty} f(t)=0 \quad \text { if } g \text { is unbounded, } \quad \lim _{t \rightarrow \infty} t f(t)=\frac{1}{b}, \quad \lim _{t \searrow 0} f(t)=\frac{1}{a},
$$

where we write $1 / \infty:=0$. In particular, we have

$$
f \in \mathrm{A}_{+}^{1}\left(\mathbb{C}_{+}\right) \quad \Longleftrightarrow \quad f(0+)<\infty \quad \Longleftrightarrow \quad g(0+)>0 .
$$

It is not always easy to identify potentials. One way is by virtue of Hirsch's theorem [26, 27] saying that $f$ is a potential if for every $t>0$ the sequence

$$
\alpha_{n}:=(-1)^{n} \frac{f^{(n)}(t)}{n !} \quad(n \geq 0)
$$

is log-convex, i.e., satisfies

$$
\alpha_{n}^{2} \leq \alpha_{n-1} \alpha_{n+1} \quad(n \geq 1) .
$$

Alternatively, a completely monotone function $f$ is a potential if

$$
f(z)=c+\int_{0}^{\infty} e^{-s z} v(s) \mathrm{d} s \quad(\operatorname{Re} z>0),
$$

where $c \geq 0$ and $v:(0, \infty) \rightarrow(0, \infty)$ is a decreasing and log-convex function [41, Theorem 10.23, Corollary 10.24]. In [31, fundamental for our paper [22, the logconvexity of a sequence is shown to be crucial for the study of inverses of functions analytic on the unit disc. Kaluza's results in 31] parallel those of Hirsch's, and in fact can be used to deduce Hirsch's theorem mentioned above.

We shall now show that Bernstein functions are always part of the extended HP-functional calculus. For a related statement see [6, Theorem 1.6]. 
Lemma 2.5. Every Bernstein function $g$ can be written in the form

$$
g(z)=g_{1}(z)+z g_{2}(z), \quad z>0,
$$

where $g_{1}, g_{2} \in \mathrm{A}_{+}^{1}\left(\mathbb{C}_{+}\right)$.

Proof. Suppose that $g \sim(a, b, \mu)$ as in (2.7). We extend naturally $\mu$ to $\mathbb{R}_{+}$by setting $\mu(\{0\})=0$ and let

$$
\nu(\mathrm{d} s):=\frac{s}{s+1} \mu(\mathrm{d} s) \in \mathrm{M}\left(\mathbb{R}_{+}\right) .
$$

Then we write

$$
\left(1-e^{-s z}\right) \frac{s+1}{s}=1-e^{-s z}+z \frac{1}{s} \int_{0}^{s} e^{-r z} \mathrm{~d} r
$$

Integrating this against $\nu$ with respect to the variable $s>0$ and using Fubini's theorem, we obtain

$$
\begin{aligned}
g(z) & -a-b z=\int_{0+}^{\infty}\left(1-e^{-s z}\right) \mu(\mathrm{d} s)=\int_{0+}^{\infty}\left(1-e^{-s z}\right) \frac{s+1}{s} \nu(\mathrm{d} s) \\
& =c-(\mathcal{L} \nu)(z)+z \int_{0}^{\infty} \frac{1}{s} \int_{0}^{s} e^{-r z} \mathrm{~d} r \nu(\mathrm{d} s) \\
& =c-(\mathcal{L} \nu)(z)+z \int_{0}^{\infty}\left[\int_{r}^{\infty} \frac{\nu(\mathrm{d} s)}{s}\right] e^{-r z} \mathrm{~d} r \\
& =c-(\mathcal{L} \nu)(z)+z(\mathcal{L} \gamma)(z)
\end{aligned}
$$

with

$$
c=\nu(0, \infty) \geq 0 \quad \text { and } \quad \gamma(\mathrm{d} r)=\left[\int_{r}^{\infty} \frac{\nu(\mathrm{d} s)}{s}\right] \mathrm{d} r .
$$

Note that $\gamma$ is a positive bounded measure of total mass $\gamma\left(\mathbb{R}_{+}\right)=c$.

As a consequence we obtain that every Bernstein function is regularizable by any of the functions $e_{\lambda}(z)=(\lambda+z)^{-1}, \operatorname{Re} \lambda>0$ (corresponding to the resolvents $\left.(\lambda+A)^{-1}\right)$.

Corollary 2.6. Let $-A$ generate a bounded $C_{0}$-semigroup $(T(s))_{s \geq 0}$ on a Banach space $X$, and let $g \sim(a, b, \mu)$ be a Bernstein function. Then $g(A)$ is defined in the extended HP-functional calculus. Moreover, $\operatorname{dom}(A) \subseteq \operatorname{dom}(g(A))$ and

$$
g(A) x=a x+b A x+\int_{0+}^{\infty}(I-T(s)) x \mu(\mathrm{d} s)
$$

for each $x \in \operatorname{dom}(A)$, and $\operatorname{dom}(A)$ is a core for $g(A)$. If $A x=0$, then $g(A) x=a x$, and if $a>0$, then $\operatorname{ran}(g(A))=X$ and $g(A)$ is invertible.

Proof. That $g(A)$ is well defined follows immediately from Lemma 2.5, To prove the formula for $x \in \operatorname{dom}(A)$, we insert $A$ in the representation of $g$ derived in the proof of Lemma 2.5 (using the definition of $g(A)$ via regularization) and obtain

$$
g(A) x-a x-b A x=c x-\int_{0}^{\infty} T(s) x \nu(\mathrm{d} s)+\int_{0}^{\infty} T(s) A x \gamma(\mathrm{d} s) .
$$

Then we reverse the computation in the proof of Lemma 2.5 using the formula

$$
x-T(s) x=\int_{0}^{s} T(r) A x \mathrm{~d} r \quad(x \in \operatorname{dom}(A))
$$


to arrive at (2.9). If $a>0$ then $f=1 / g \in \mathrm{A}_{+}^{1}\left(\mathbb{C}_{+}\right)$, hence $f g=1$ and $f(A) g(A) \subseteq$ $g(A) f(A)=I$. The remaining statement follows from Lemma 2.1 with $n=1$.

Remark 2.7. The formula (2.9) has been first obtained by Phillips in [37. In his approach, the operator that we now denote by $-g(A)$ is defined as the generator of a certain semigroup subordinate in the sense of Bochner to the semigroup whose generator is $-A$. Curiously enough, Phillips does not use the notation " $g(A)$ ", not even informally. Balakrishnan 2 gave the first definition of a proper unbounded functional calculus that extends the Hille-Phillips calculus. His construction covers subordinate semigroups, as he explains in [2, Section 5], but the formula (2.9) is not explicitly treated. Schilling [40, probably unaware of Balakrishnan's paper, gives an alternative description of $g(A)$ for $g$ a complete Bernstein function, and uses it to prove functional calculus properties such as the product rule and a composition rule, see also [41, Chapter 12]. The general method of regularization that we use to extend the Hille-Phillips calculus, was first described in full generality by deLaubenfels in [15] as "Construction Two", but is modelled on earlier work of McIntosh and Bade, see [23, Sections 2.8 and 4.6]. Schilling cites deLaubenfels' paper in the bibliography, but does not relate his functional calculus to deLaubenfels' approach. This has been achieved now with our Lemma 2.5] and Corollary 2.6.

Complete and Special Bernstein Functions. A Bernstein function is called a complete Bernstein function if its representing measure has a completely monotone density with respect to Lebesgue measure, see [41, Definition 6.1]. A Bernstein function $g \neq 0$ is called special if $z / g(z)$ is again a Bernstein function. By [41, Proposition 7.1], if $g \neq 0$ is a complete Bernstein function, then so is $z / g(z)$. Hence every non-zero complete Bernstein function is special.

By [41, Theorem 10.3, Remark 10.4, (ii)], a Bernstein function $g \neq 0$ is special if and only if the associated potential $f=1 / g$ has the Laplace transform representation

$$
f(z)=c+\int_{0}^{\infty} e^{-s z} v(s) \mathrm{d} s \quad(\operatorname{Re} z>0)
$$

where $c \geq 0$ and $v:(0, \infty) \rightarrow(0, \infty)$ is decreasing. Building on this, we can prove that $|g(z)| \sim g(|z|)$, a fact of independent interest that will be important in Section 4 below. More precisely, we have the following result.

Theorem 2.8. Let $g \neq 0$ be a special Bernstein function. Then

$$
\frac{1}{3 e}|g(z)| \leq g(|z|) \leq 3 e|g(z)|
$$

for all $z$ with $\operatorname{Re} z \geq 0$.

Proof. By continuity of $g$ in the closed right half-plane, it suffices to prove (2.11) only for $\operatorname{Re} z>0$. Let $f:=1 / g$, and $c, v$ as in (2.10). We first establish the right-hand inequality, which is equivalent to

$$
|f(z)| \leq 3 \operatorname{eef}(|z|) \quad(\operatorname{Re} z>0) .
$$


Employing integration by parts for (improper) Riemann-Stieltjes integrals [25. Theorem 3.3.1], we compute with $t, \operatorname{Re} z>0$ :

$$
\begin{aligned}
f(z) & =c+\int_{0}^{t} e^{-z s} v(s) \mathrm{d} s-\frac{1}{z} \int_{t}^{\infty} v(s) \mathrm{d} e^{-z s} \\
& =c+\int_{0}^{t} e^{-z s} v(s) \mathrm{d} s-\left.\frac{v(s) e^{-z s}}{z}\right|_{s=t} ^{s=\infty}+\frac{1}{z} \int_{t}^{\infty} e^{-s z} \mathrm{~d} v(s) \\
& =c+\int_{0}^{t} e^{-z s} v(s) \mathrm{d} s+\frac{v(t) e^{-z t}}{z}+\frac{1}{z} \int_{t}^{\infty} e^{-s z} \mathrm{~d} v(s) .
\end{aligned}
$$

Hence, since $v$ is decreasing,

$$
\begin{aligned}
|f(z)| & \leq c+\int_{0}^{t} v(s) \mathrm{d} s+\frac{v(t) e^{-t \operatorname{Re} z}}{|z|}-\frac{1}{|z|} \int_{t}^{\infty} e^{-s \operatorname{Re} z} \mathrm{~d} v(s) \\
& \leq c+\int_{0}^{t} v(s) \mathrm{d} s+\frac{e^{-t \operatorname{Re} z}}{|z|}\left(v(t)-\int_{t}^{\infty} \mathrm{d} v(s)\right) \\
& \leq c+\int_{0}^{t} v(s) \mathrm{d} s+\frac{2 v(t)}{|z|} \leq c+\left(1+\frac{2}{t|z|}\right) \int_{0}^{t} v(s) \mathrm{d} s \\
& \leq c+\left(1+\frac{2}{t|z|}\right) e^{t|z|} \int_{0}^{t} e^{-s|z|} v(s) \mathrm{d} s \leq\left(1+\frac{2}{t|z|}\right) e^{t|z|} f(|z|) .
\end{aligned}
$$

Now we insert $t=1 /|z|$ and arrive at (2.12), concluding the proof of the right-hand inequality in (2.11).

The left-hand inequality follows immediately be applying the right-hand inequality to the special Bernstein function $z / g(z)$.

Stieltjes Functions. A function $f:(0, \infty) \rightarrow \mathbb{R}_{+}$is called a Stieltjes function if it can be written as

$$
f(z)=\frac{a}{z}+b+\int_{0+}^{\infty} \frac{\mu(\mathrm{d} s)}{z+s} \quad(z>0)
$$

where $a, b \geq 0$ and $\mu$ is a positive Radon measure on $(0, \infty)$ satisfying

$$
\int_{0+}^{\infty} \frac{\mu(\mathrm{d} s)}{1+s}<\infty
$$

In this case, $\mu$ is called a Stieltjes measure and (2.13) is called the Stieltjes representation for $f$, since such a representation is unique, see [41, Chapter 2].

Example 2.9. We show that the function

$$
f(z):=\frac{\log z}{z-1}
$$

is a Stieltjes function. (Here and in the following, $\log z$ denotes the principal branch of the complex logarithm.) To this aim, we depart from the representation

$$
\log (1+z)=\int_{0}^{\infty}\left(1-e^{-s z}\right) e^{-s} \frac{\mathrm{d} s}{s}
$$

valid for $\operatorname{Re} z>-1$. (To see this, just take the derivative of the right-hand side with respect to $z$.) By changing $z$ to $z-1$ we obtain

$$
\log z=\int_{0}^{\infty}\left(1-e^{-s(z-1)}\right) e^{-s} \frac{\mathrm{d} s}{s}
$$


valid for $\operatorname{Re} z>0$. Hence

$$
\begin{aligned}
\frac{\log z}{z-1} & =\int_{0}^{\infty} \frac{1-e^{-s(z-1)}}{z-1} e^{-s} \frac{\mathrm{d} s}{s}=\int_{0}^{\infty} \int_{0}^{s} e^{-t(z-1)} \mathrm{d} t e^{-s} \frac{\mathrm{d} s}{s} \\
& =\int_{0}^{\infty} \int_{t}^{\infty} \frac{e^{-(s-t)}}{s} \mathrm{~d} s e^{-t z} \mathrm{~d} t=\int_{0}^{\infty}\left[\int_{0}^{\infty} \frac{e^{-s}}{s+t} \mathrm{~d} s\right] e^{-t z} \mathrm{~d} t .
\end{aligned}
$$

A change of variable $s \mapsto z s$ (with $z>0$ ) and $t \mapsto s t$ leads to

$$
\begin{aligned}
\frac{\log z}{z-1} & =\int_{0}^{\infty}\left[\int_{0}^{\infty} \frac{z e^{-s z}}{s z+t} \mathrm{~d} s\right] e^{-t z} \mathrm{~d} t=\int_{0}^{\infty} \int_{0}^{\infty} \frac{z s e^{-s(z+t z)}}{s(z+t)} \mathrm{d} s \mathrm{~d} t \\
& =\int_{0}^{\infty} \frac{z}{z+z t} \frac{\mathrm{d} t}{z+t}=\int_{0}^{\infty} \frac{1}{z+t} \frac{\mathrm{d} t}{1+t}
\end{aligned}
$$

and this is a Stieltjes representation for $f$.

Stieltjes functions are interesting in our context because of the following result [11, Proposition 7.1 and Theorem 7.3].

Theorem 2.10. The function $f \neq 0$ is a Stieltjes function if and only if $1 / f$ is a complete Bernstein function if and only if $z f(z)$ is a complete Bernstein function. In particular, every non-zero Stieltjes function is a potential.

In particular, by Example 2.9 above, the function

$$
f(z)=\frac{\log z}{z-1}
$$

is a potential, and $1 / f$ is a complete Bernstein function. This fact will be used in the following.

The next result gives a useful characterization of Stieltjes functions 41, Theorem 6.2 and Corollary 7.4].

Theorem 2.11. A non-zero function $f$ is a Stieltjes function if and only if $f$ admits an analytic extension to $\mathbb{C} \backslash(-\infty, 0]$ such that

$$
\begin{array}{ll} 
& f(0+):=\lim _{t \searrow 0} f(t) \text { exists, } \quad f(0+) \in(0, \infty], \\
\text { and } & \operatorname{Im} z \cdot \operatorname{Im} f(z) \leq 0 \quad \text { for all } z \notin(-\infty, 0] .
\end{array}
$$

Note that if $g(z)=\log (1+z)$ then

$$
\frac{g(z)-g(1 / z)}{z-1}=\frac{\log (1+z)-\log (1+1 / z)}{z-1}=\frac{\log z}{z-1}
$$

and, since

$$
\frac{e^{-s}}{s}=\int_{1}^{\infty} e^{-s \tau} \mathrm{d} \tau
$$

$\log (1+z)$ is a complete Bernstein function, by (2.14). Hence the result of Example 2.9 is actually a consequence of the following theorem.

Theorem 2.12. Let $g \neq 0$ be a complete Bernstein function with $\lim _{t} \succ_{\infty} g(t) / t=$ 0. Then

$$
f(z):=\frac{g(z)-g(1 / z)}{z-1}
$$

is a Stieltjes function, hence a potential. 
Proof. By Theorem 2.11 the function $t \mapsto g(t) / t$ is a Stieltjes function. Since $\lim _{t \rightarrow \infty} g(t) / t=0$, we have

$$
g(z)=a+\int_{0+}^{\infty} \frac{z}{z+s} \rho(\mathrm{d} s) \quad(z>0)
$$

with $a \geq 0$ and the representing measure $\rho$ satisfying $\int_{0+}^{\infty} \frac{\rho(\mathrm{d} s)}{1+s}<\infty$. Hence

$$
f(z)=\frac{1}{z-1} \int_{0+}^{\infty}\left[\frac{z}{z+s}-\frac{1}{1+z s}\right] \rho(\mathrm{d} s)=\int_{0+}^{\infty} \frac{(z+1) s \rho(\mathrm{d} s)}{(1+z s)(z+s)} \quad(z>0) .
$$

So, the function $f:(0, \infty) \rightarrow(0, \infty)$ extends analytically into $\mathbb{C} \backslash(-\infty, 0]$. Moreover, for $s>0$ and $z \in \mathbb{C}, z \neq-s,-1 / s$ we have

$$
\begin{array}{r}
|1+z s|^{2}|z+s|^{2} \frac{(z+1)}{(z+s)(1+z s)}=(z+1)(1+\bar{z} s)(s+\bar{z}) \\
=s+\left(s^{2}+1\right)|z|^{2}+\bar{z}\left(s^{2}+1+s|z|^{2}\right)+s \bar{z}^{2}+s z .
\end{array}
$$

Taking imaginary parts, we obtain

$$
\begin{aligned}
|1+z s|^{2} & |z+s|^{2} \operatorname{Im} \frac{(z+1)}{(z+s)(1+z s)} \\
& =-(\operatorname{Im} z)\left(s^{2}+1+s|z|^{2}\right)+(\operatorname{Im} z) s-2 s(\operatorname{Im} z)(\operatorname{Re} z) \\
& =-(\operatorname{Im} z)\left(s^{2}+1+s|z|^{2}-s+2 s \operatorname{Re} z\right) \\
& =-(\operatorname{Im} z)\left((s-1)^{2}+s\left(|z|^{2}+2 \operatorname{Re} z+1\right)\right)
\end{aligned}
$$

Since $|z|^{2}+2 \operatorname{Re} z+1 \geq(1+\operatorname{Re} z)^{2} \geq 0$ for all $z \in \mathbb{C}$, we see that

$$
\operatorname{Im} z \cdot \operatorname{Im} f(z)=\frac{-(\operatorname{Im} z)^{2}\left((s-1)^{2}+s\left(|z|^{2}+2 \operatorname{Re} z+1\right)\right)}{|1+z s|^{2}|z+s|^{2}} \leq 0 .
$$

whenever $z \in \mathbb{C}$ and $z \neq-s,-1 / s$. Furthermore,

$$
\begin{aligned}
\frac{\mathrm{d}}{\mathrm{d} t}\left(\frac{(t+1)}{(1+t s)(t+s)}\right) & =\frac{(1+t s)(t+s)-(1+t)\left(s^{2}+2 t s+1\right)}{(1+t s)^{2}(t+s)^{2}} \\
& =\frac{-(s-1)^{2}-s(1+t)^{2}}{(1+t s)^{2}(t+s)^{2}} \leq 0
\end{aligned}
$$

for $t>0$, and hence $\lim _{t \backslash 0} f(t)$ exists and belongs to $(0, \infty]$. By Theorem 2.11 again, $f$ is a Stieltjes function.

\section{Estimating Rates in Terms of the Pre-Laplace Transform}

For $t>0$ we define

$$
\mathrm{C}_{t}(z):=\frac{1}{t} \int_{0}^{t} e^{-s z} \mathrm{~d} s=\frac{1-e^{-t z}}{t z} \quad(\operatorname{Re} z \geq 0) .
$$

Then $\mathrm{C}_{t}(z)=\mathrm{C}_{1}(t z) \in \mathrm{A}_{+}^{1}\left(\mathbb{C}_{+}\right)$as well as $z \mathrm{C}_{t}(z) \in \mathrm{A}_{+}^{1}\left(\mathbb{C}_{+}\right)$, with

$$
\sup _{t>0}\left\|\mathrm{C}_{t}\right\|_{\mathrm{A}_{+}^{1}}+\sup _{t>0}\left\|t z \mathrm{C}_{t}\right\|_{\mathrm{A}_{+}^{1}}<\infty .
$$

For a Bernstein function $g \sim(a, b, \mu)$ we define

$$
r(t)=r[g](t):=\frac{a}{2}+\frac{b}{t}+\int_{0+}^{\infty} \min (s / t, 1) \mu(\mathrm{d} s) \quad(t>0) .
$$


Note that by Fubini's theorem

$$
\int_{0+}^{\infty} \min (s / t, 1) \mu(\mathrm{d} s)=\frac{1}{t} \int_{0}^{t} \mu(r, \infty) \mathrm{d} r \quad(t>0) .
$$

The following theorem is the reason why we are interested in the function $r(t)$.

Theorem 3.1. Let $g \sim(a, b, \mu)$ be a Bernstein function and $r=r[g]$ as above. Then $\mathrm{C}_{t} g \in \mathrm{A}_{+}^{1}\left(\mathbb{C}_{+}\right)$for each $t>0$ and

$$
\left\|\mathrm{C}_{t} g\right\|_{\mathrm{A}_{+}^{1}}=2 r(t) \quad(t>0) .
$$

Proof. Suppose first that $a=0$. Applying Fubini's theorem twice we compute

$$
\begin{aligned}
t \mathrm{C}_{t}(z) g(z) & =\int_{0}^{t} e^{-r z} \mathrm{~d} r\left(b z+\int_{0+}^{\infty}\left(1-e^{-s z}\right) \mu(\mathrm{d} s)\right) \\
& =b\left(1-e^{-t z}\right)+\int_{0}^{t} \int_{0+}^{\infty}\left(e^{-z r}-e^{-z(r+s)}\right) \mu(\mathrm{d} s) \mathrm{d} r \\
& =b\left(1-e^{-t z}\right)+\int_{0+}^{\infty}\left(\int_{0}^{t} e^{-z r} \mathrm{~d} r-\int_{s}^{s+t} e^{-z r} \mathrm{~d} r\right) \mu(\mathrm{d} s) \\
& =b\left(1-e^{-t z}\right)+\int_{0+}^{\infty}\left(\int_{0}^{\min (s, t)} e^{-z r} \mathrm{~d} r-\int_{\max (s, t)}^{s+t} e^{-z r} \mathrm{~d} r\right) \mu(\mathrm{d} s) \\
& =b\left(1-e^{-t z}\right)+\int_{0}^{t} \mu(r, \infty) e^{-r z} \mathrm{~d} r-\int_{t}^{\infty} \mu(r-t, r) e^{-z r} \mathrm{~d} r .
\end{aligned}
$$

This is the Laplace transform of the measure $b \delta_{0}-b \delta_{t}+\psi(r) \mathrm{d} r$, where

$$
\psi(r)=\chi_{[0, t]}(r) \mu(r, \infty)-\chi_{(t, \infty)}(r) \mu(r-t, r), \quad\left(r \in \mathbb{R}_{+}\right) .
$$

Hence we see that

$$
\left\|t \mathrm{C}_{t} g\right\|_{\mathrm{A}_{+}^{1}}=2 b+\int_{0}^{t} \mu(r, \infty) \mathrm{d} r+\int_{t}^{\infty} \mu(r-t, r) \mathrm{d} r
$$

However, since $g(0+)=0$, the two integrals here must be equal, and hence

$$
\left\|t \mathrm{C}_{t} g\right\|_{\mathrm{A}_{+}^{1}}=2 b+2 \int_{0}^{t} \mu(r, \infty) \mathrm{d} r=2 \operatorname{tr}(t)
$$

as claimed. If $a>0$ then we have to add the term $a \int_{0}^{t} e^{-r z} \mathrm{~d} r$ in each line of the computation from above. This leads to the representation

$$
t \mathrm{C}_{t}(z) g(z)=b\left(1-e^{-t z}\right)+\int_{0}^{t}(a+\mu(r, \infty)) e^{-r z} \mathrm{~d} r-\int_{t}^{\infty} \mu(r-t, r) e^{-z r} \mathrm{~d} r
$$

and hence to the norm identity

$$
\left\|t \mathrm{C}_{t} g\right\|_{\mathrm{A}_{+}^{1}}=a t+2 b+2 \int_{0}^{t} \mu(r, \infty) \mathrm{d} r=2 \operatorname{tr}(t)
$$

The next result lists some properties of the function $r[g]$, and answers the question which functions $r$ on $(0, \infty)$ can arise as $r=r[g]$.

Theorem 3.2. For a function $r:(0, \infty) \rightarrow(0, \infty)$ the following assertions are equivalent. 
(i) There exists a Bernstein function $0 \neq g \sim(a, b, \mu)$ such that $r=r[g]$.

(ii) The function $t \mapsto \operatorname{tr}(t)$ is strictly positive, increasing and concave.

Moreover, if (i) or (ii) is satisfied, then the following assertions hold.

a) $r$ is continuous and decreasing, with $\lim _{t \rightarrow \infty} r(t)=a / 2$.

b) $\lim _{t \searrow 0} \operatorname{tr}(t)=b$.

c) The function $t \mapsto \operatorname{tr}(t)$ is bounded if and only if $a=0$ and there is $h \in \mathrm{A}_{+}^{1}\left(\mathbb{C}_{+}\right)$ such that $g(z)=z h(z)$. In this case $h$ is completely monotone.

Proof. (i) implies (ii): Let $g \sim(a, b, \mu)$. Then

$$
r(t)=a / 2+b / t+\int_{0+}^{\infty} \min (s / t, 1) \mu(\mathrm{d} s)
$$

is decreasing on $(0, \infty)$, continuous, and satisfies $\lim _{t \rightarrow \infty} r(t)=a / 2$ by the monotone convergence theorem. Furthermore, the function

$$
f(t):=\operatorname{tr}(t)=t a / 2+b+\int_{0}^{t} \mu(r, \infty) \mathrm{d} r
$$

is increasing on $(0, \infty)$ and satisfies $\lim _{t \searrow_{0}} f(t)=b$. Since the last summand is an integral of a decreasing positive function, it is concave, and hence so is $f$. Note that $f$ has one-sided derivatives

$$
D_{+} f(t)=\frac{a}{2}+\mu(t, \infty) \quad \text { and } \quad D_{-} f(t)=\frac{a}{2}+\mu[t, \infty) \quad(t>0) .
$$

(ii) implies (i): Define $f(t):=\operatorname{tr}(t)$. Since $f$ is concave, $f$ is absolutely continuous, has a right derivative $D_{+} f(t)$ at each $t>0$, and the function $D_{+} f$ is decreasing and right continuous. Since $f$ is increasing, $D_{+} f(t) \geq 0$ for all $t>0$. Define $a:=2 \lim _{t \rightarrow \infty} D_{+} f(t)$. Then the function $m(t):=D_{+} f(t)-(a / 2)$ is positive, right continuous and decreases to 0 . By standard measure theory there exists a positive Radon measure $\mu$ on $(0, \infty)$ satisfying $m(t)=\mu(t, \infty)$ for all $t>0$.

Since $f$ is absolutely continuous, we have

$$
f(t)-f(s)=\int_{s}^{t} D_{+} f(r) \mathrm{d} r=\frac{a}{2}(t-s)+\int_{s}^{t} \mu(r, \infty) \mathrm{d} r
$$

for all $0<s<t<\infty$. Letting $s \rightarrow 0+$ here we obtain

$$
f(t)=b+\frac{a}{2} t+\int_{0}^{t} \mu(r, \infty) \mathrm{d} r
$$

for any $t>0$, where $b:=\lim _{t \searrow 0} f(t)$. In particular $\int_{0}^{1} \mu(r, \infty) \mathrm{d} r<\infty$, which implies that

$$
\int_{0+}^{\infty} \min (1, s) \mu(\mathrm{d} s)<\infty .
$$

Hence $r(t)=f(t) / t=r[g](t)$ for the Bernstein function $g \sim(a, b, \mu)$.

It remains to show c). Clearly $\operatorname{tr}(t)$ is bounded on $(0, \infty)$ if and only if $a=0$ and

$$
\int_{0}^{\infty} \mu(r, \infty) \mathrm{d} r=\int_{0+}^{\infty} s \mu(\mathrm{d} s)<\infty
$$


On the other hand, by Fubini's theorem,

$$
g(z)=b z+\int_{0+}^{\infty}\left(1-e^{-r z}\right) \mu(\mathrm{d} s)=b z+z \int_{0}^{\infty} \mu(r, \infty) e^{-r z} \mathrm{~d} r=z h(z),
$$

where $h$ is the Laplace transform of the positive measure $\nu(\mathrm{d} r):=b \delta_{0}(\mathrm{~d} r)+$ $\mu(r, \infty) \mathrm{d} r$. The measure $\nu$ is finite if and only if (3.3) holds. The claimed equivalence now follows from the injectivity of the Laplace transform.

Remark 3.3. See TheoremB.3 below for a related result on rate functions associated with complete Bernstein functions.

We now employ the functional calculus. Let $-A$ be the generator of a bounded semigroup $(T(s))_{s \geq 0}$, and let $M:=\sup _{s \geq 0}\|T(s)\|$. If $g$ is a Bernstein function then, by Theorem 3.1 and (2.2), we have

$$
\left\|g(A) \mathrm{C}_{t}(A)\right\| \leq 2 M r(t) \quad(t>0) .
$$

Hence by (2.3) for $y=g(A) x \in \operatorname{ran}(g(A))$,

$$
\left\|\mathrm{C}_{t}(A) y\right\|=\left\|g(A) \mathrm{C}_{t}(A) x\right\| \leq 2 M r(t)\|x\| \quad(t>0) .
$$

By the monotone convergence theorem, $\lim _{t \rightarrow \infty} 2 r(t)=g(0+)$. Hence, if $g(0+)>0$ then nothing is gained. This is no surprise since we have seen above that in this case $\operatorname{ran}(g(A))=X$, and there is no general convergence rate on the whole space. However, in the case $g(0+)=0$ we obtain a convergence rate to zero. Let us summarize our considerations.

Theorem 3.4. Let $g \sim(a, b, \mu)$ be a Bernstein function, and let $r=r[g]$ be as in (3.2). Let $-A$ be the generator of a $C_{0}$-bounded semigroup $(T(s))_{s \geq 0}$ on a Banach space $X$ with $M:=\sup _{s \geq 0}\|T(s)\|$. Then the following statements hold.

a) For each $y=g(A) x$

$$
\left\|\mathrm{C}_{t}(A) y\right\| \leq 2 M r(t)\|x\| \quad(t>0) .
$$

b) If $\operatorname{tr}(t) \rightarrow \infty$ as $t \rightarrow \infty, g(0+)=0$ and $(T(s))_{s \geq 0}$ is mean ergodic, then

$$
\left\|\mathrm{C}_{t}(A) y\right\|=\mathrm{o}(r(t)) \quad \text { as } t \rightarrow \infty
$$

whenever $y \in \operatorname{ran}(g(A))$.

c) If $\operatorname{tr}(t)=\mathrm{O}(1)$ as $t \rightarrow \infty$, then $\operatorname{ran}(g(A)) \subseteq \operatorname{ran}(A)$ and

$$
\left\|\mathrm{C}_{t}(A) y\right\|=\mathrm{O}\left(t^{-1}\right) \quad \text { as } t \rightarrow \infty
$$

whenever $y \in \operatorname{ran}(g(A))$.

Proof. The estimate (3.5) was obtained above. To prove b), suppose that $a=$ $g(0+)=0, \operatorname{tr}(t) \rightarrow \infty$ as $t \rightarrow \infty$, and that the semigroup $(T(s))_{s \geq 0}$ is mean ergodic. By (3.4), the family of operators

$$
S_{t}:=r(t)^{-1} g(A) \mathrm{C}_{t}(A), \quad t>0,
$$

is uniformly bounded. If $A x=0$ then $g(A) x=a x=0$ (Corollary 2.6) and hence $S_{t} x=0$ for $t>0$. On the other hand, if $x \in \operatorname{dom}(A) \subseteq \operatorname{dom}(g(A))$ then $y=A x \in$ $\operatorname{ran}(A)$ and using (2.3) we obtain

$$
S_{t} y=r(t)^{-1} g(A) \mathrm{C}_{t}(A) A x=\frac{1}{\operatorname{tr}(t)}\left[t A \mathrm{C}_{t}(A)\right] g(A) x=\frac{1}{\operatorname{tr}(t)}\left[t z \mathrm{C}_{t}(z)\right](A) g(A) x .
$$


Taking norms we infer that

$$
\left\|S_{t} y\right\| \leq \frac{M\left\|t z \mathrm{C}_{t}\right\|_{\mathrm{A}_{+}^{1}}}{\operatorname{tr}(t)}\|g(A) x\| \rightarrow 0 \quad \text { as } t \rightarrow \infty
$$

by (3.1). Since the semigroup is mean ergodic, $\operatorname{ker}(A) \oplus \operatorname{ran}(A)$ is dense in $X$, and hence $S_{t} \rightarrow 0$ strongly on $X$. It remains to note that for $y=g(A) x$ one has $S_{t} y=r(t)^{-1} \mathrm{C}_{t}(A) g(A) x$ as above.

For the proof of c) suppose now that $\operatorname{tr}(t)$ stays bounded as $t \rightarrow \infty$. Then by c) of Theorem 3.2 we have $g(z)=z h(z)$ for some $h \in \mathrm{A}_{+}^{1}\left(\mathbb{C}_{+}\right)$, and hence $g(A)=A h(A)$ by the functional calculus (see (2.3) ). This implies that $\operatorname{ran}(g(A)) \subseteq \operatorname{ran}(A)$, and then $y=A x$ for some $x \in \operatorname{dom}(A)$. Thus $\mathrm{C}_{t}(A) y=t^{-1}(x-T(t) x), t>0$, so that $\left\|\mathrm{C}_{t}(A) y\right\|=\mathrm{O}\left(t^{-1}\right)$ as $t \rightarrow \infty$ (cf. Proposition 1.1 b)).

As a direct consequence of Theorems 3.2 and 3.4 we state the following corollary.

Corollary 3.5. Let $r:(0, \infty) \rightarrow(0, \infty)$ be such that $t \mapsto t r(t)$ is strictly positive, increasing and concave. Then there is a Bernstein function $g$ such that $r=r[g]$, and hence the conclusion of Theorem 3.4 holds.

Thus, in particular, any function $r$ subject to the condition (ii) of Theorem 3.4 can be realized as a rate of decay of $\mathrm{C}_{t}(A)$ restricted to the range of $g(A)$.

\section{Estimating Rates in Terms of Laplace Transforms}

So far, the rate $r$ is given in terms of the measure $\mu$ from the representation (2.7) of the Bernstein function $g$. However, in situations of interest we often only know the measure $\nu$ corresponding to a potential $f=1 / g$, and so it seems desirable to be able to read off $r$ (or at least its asymptotic behaviour) from the values of $f$ at certain points.

To achieve this, we begin with some elementary considerations involving the simple inequalities

$$
\begin{aligned}
x e^{-x} & \leq \min \left(2, x, 1-e^{-x}\right) & & (x>0), \\
\left|1-e^{-z s}\right| & \leq \min (2, s|z|) & & (s, \operatorname{Re} z \geq 0) .
\end{aligned}
$$

Lemma 4.1. Let $g \sim(a, b, \mu)$ be a Bernstein function, and $r:=r[g]$. Then

$$
\left[(t \operatorname{Re} z) e^{-t \operatorname{Re} z}\right] r(t) \leq \operatorname{Re} g(z) \leq|g(z)| \leq \max (2, t|z|) r(t)
$$

for all $t>0$ and $z \in \mathbb{C}$ with $\operatorname{Re} z>0$.

Proof. We have

$$
\begin{aligned}
|g(z)| & \leq a+b|z|+\int_{0+}^{\infty}\left|1-e^{-s z}\right| \mu(\mathrm{d} s) \\
& \leq \frac{a}{2} 2+b|z|+\int_{0+}^{t} s|z| \mu(\mathrm{d} s)+\int_{t+}^{\infty} 2 \mu(\mathrm{d} s) \leq \max (2, t|z|) r(t)
\end{aligned}
$$


for the upper estimate. For the lower estimate we write $x:=t \operatorname{Re} z>0$. Then

$$
\begin{aligned}
\operatorname{Re} g(z) & \geq a+b \operatorname{Re} z+\int_{0+}^{\infty}\left(1-\operatorname{Re} e^{-z s}\right) \mu(\mathrm{d} s) \\
& \geq a+b \operatorname{Re} z+\int_{0+}^{\infty}\left(1-e^{-s \operatorname{Re} z}\right) \mu(\mathrm{d} s) \\
& \geq a+b \operatorname{Re} z+\int_{0+}^{t}(s \operatorname{Re} z) e^{-s \operatorname{Re} z} \mu(\mathrm{d} s)+\left(1-e^{-t \operatorname{Re} z}\right) \int_{t+}^{\infty} \mu(\mathrm{d} s) \\
& \geq 2 \frac{a}{2}+\frac{b}{t} x+x e^{-x} \int_{0+}^{t} s / t \mu(\mathrm{d} s)+\left(1-e^{-x}\right) \mu(t, \infty) \geq x e^{-x} r(t) .
\end{aligned}
$$

As a consequence we find that one can read off the (asymptotics of the) rate $r(t)$ from values $g\left(z_{t}\right)$ if the set $\left(z_{t}\right)_{t>0}$ is carefully chosen.

Proposition 4.2. Given $0<\alpha \leq \beta<\infty$ there are positive numbers $c_{0}=$ $c_{0}(\alpha, \beta), c_{1}=c_{1}(\alpha, \beta)$ such that the following holds. Suppose that $g$ is a Bernstein function with associated rate function $r=r[g]$. Then

$$
c_{0} r(t) \leq|g(z)| \leq c_{1} r(t)
$$

whenever $t>0$ and $\operatorname{Re} z>0$ are such that $\alpha \leq t \operatorname{Re} z \leq t|z| \leq \beta$.

Proof. By Lemma 4.1 we can choose $c_{0}(\alpha, \beta)=\inf _{\alpha \leq x \leq \beta}\left(x e^{-x}\right) \quad$ and $\quad c_{1}(\alpha, \beta)=$ $\max (2, \beta)$.

For special Bernstein functions $g$ we can employ Theorem 2.8 and obtain a better result.

Proposition 4.3. Given $0<\alpha \leq \beta<\infty$ there are positive numbers $c_{0}=$ $c_{0}(\alpha, \beta), c_{1}=c_{1}(\alpha, \beta)$ such that the following holds. If $g$ is a special Bernstein function with associated rate function $r:=r[g]$, then

$$
c_{0} r(t) \leq|g(z)| \leq c_{1} r(t)
$$

whenever $t>0$ and $\operatorname{Re} z \geq 0$ are such that $t|z| \in[\alpha, \beta]$. In particular, $c_{0}=1 /\left(3 e^{2}\right)$ if $\alpha=\beta=1$.

Proof. Combining Theorem 2.8 with Lemma 4.1 we obtain

$$
\left(\frac{t|z| e^{-t|z|}}{3 e}\right) r(t) \leq|g(z)| \leq \max (2, t|z|) r(t) \quad(t>0, \operatorname{Re} z \geq 0) .
$$

Then choose $c_{0}=(3 e)^{-1} \inf _{\alpha \leq x \leq \beta}\left(x e^{-x}\right)$ and $c_{1}=\max (2, \beta)$.

For a potential function $f$, we can take $e=1 / f$ in Theorem 2.3 and combining it with Theorem 3.4 and Proposition 4.2 we obtain the following statement.

Theorem 4.4. Let $\mu$ be a positive Radon measure on $\mathbb{R}_{+}$and let $f=\mathcal{L} \mu$ be a potential function (e.g., a Stieltjes function) with $f(0+)=\infty$. Let $-A$ be the generator of a bounded $C_{0}$-semigroup $(T(s))_{s \geq 0}$ on a Banach space $X$, and let $x, y \in X$ be such that

Then

$$
\lim _{\alpha \searrow 0} \int_{0}^{\infty} e^{-\alpha s} T(s) x \mu(\mathrm{d} s)=y \quad \text { weakly } .
$$


a) $x=(1 / f)(A) y \quad$ and $\quad\left\|\mathrm{C}_{t}(A) x\right\|=\mathrm{O}\left(\frac{1}{f(1 / t)}\right) \quad$ as $t \rightarrow \infty$.

b) $\left\|\mathrm{C}_{t}(A) x\right\|=\mathrm{o}\left(\frac{1}{f(1 / t)}\right) \quad$ as $t \rightarrow \infty$ if in addition $t / f(1 / t) \rightarrow \infty$ as $t \rightarrow \infty$.

Proof. We let $g:=1 / f$ and apply Corollary 2.4 to conclude that $x=g(A) y$. Then Theorem 3.4 and Proposition 4.2 imply that

$$
\left\|\mathrm{C}_{t}(A) x\right\|=\mathrm{O}(g(1 / t))=\mathrm{O}\left(\frac{1}{f(1 / t)}\right) \quad \text { as } t \rightarrow \infty .
$$

Now suppose that $t / f(1 / t) \rightarrow \infty$ as $t \rightarrow \infty$. In this case, by Proposition 4.2, $\operatorname{tr}(t) \rightarrow \infty$ as $t \rightarrow \infty$. Furthermore, $x \in Y:=\overline{\operatorname{ran}}(A)$ and $Y$ is $(T(s))_{s \geq 0}$-invariant, so $y \in Y$ as well. This means that we can suppose without loss of generality that $(T(s))_{s \geq 0}$ is mean ergodic. Hence the second part of Theorem 3.4 yields that

$$
\left\|\mathrm{C}_{t}(A) x\right\|=\mathrm{o}(r(t))=\mathrm{o}(1 / f(1 / t)) \quad \text { as } t \rightarrow \infty,
$$

again by Proposition 4.2

Remark 4.5. Let $f$ be a Stieltjes function with the representation (cf. (2.13)

$$
f(z)=\int_{0+}^{\infty} \frac{\mu(\mathrm{d} s)}{s+z}, \quad(z>0), \quad \text { with } \quad \int_{0+}^{\infty} \frac{\mu(\mathrm{d} s)}{s+1}<\infty .
$$

Then

$$
f(z)=\int_{0}^{\infty} e^{-z t} \int_{0+}^{\infty} e^{-t s} \mu(\mathrm{d} s) \mathrm{d} t=\int_{0}^{\infty} e^{-z t} m(t) \mathrm{d} t,
$$

where $m$ is a completely monotone function such that

$$
\int_{0}^{1} m(t) \mathrm{d} t<\infty \quad \text { and } \quad \lim _{t \rightarrow \infty} m(t)=0 .
$$

Hirsch proved in [28, Corollaire, p. 214-215] that if $\operatorname{ran}(A)$ is dense in $X$, the following statements are equivalent for $x \in X$ :

(i) weak $\lim _{\alpha \searrow 0} \int_{0}^{\infty} e^{-\alpha s} T(s) x m(s) \mathrm{d} s$ exists;

(ii) $\lim _{\alpha \searrow 0} \int_{0}^{\infty} e^{-\alpha s} T(s) x m(s) \mathrm{d} s$ exists;

(iii) weak $\lim _{M \rightarrow \infty} \int_{0}^{M} T(s) x m(s) \mathrm{d} s$ exists;

(iv) $\lim _{M \rightarrow \infty} \int_{0}^{M} T(s) x m(s) \mathrm{d} s$ exists.

Moreover, all limits in (i)-(iv) are equal to each other.

We note the following: since Stieltjes functions are potentials, we can apply Theorem 4.4 to see that (i) implies $x=g(A) y$, for $g=1 / f$, where $y$ is the limit in (i). On the other hand, $A$ is injective (since $\operatorname{ran}(A)$ is dense) and $f(z)=q(z) / z$ for some Bernstein function $q$. Hence the function $f$ belongs to the extended HPcalculus for $A$. General functional calculus rules then yield that $x \in \operatorname{dom}(f(A))$ and $f(A) x=y$ [23, Corollary 1.2.4]. The point is now that one can pass, conversely, from $x \in \operatorname{dom}(f(A))$ and $f(A) x=y$ to (iv). However, Hirsch's proof for this is based essentially on the functional calculus for sectorial operators, and is beyond the 
scope of the present article. The issue will be thoroughly addressed in a subsequent paper.

Propositions 4.2 and 4.3 are not only useful to determine $r$ from values of $g$, but also to see that under some weak spectral conditions on $A$ the rate $r$ is indeed optimal on $\operatorname{ran}(g(A))$. The following result illustrates what we mean by this.

Theorem 4.6. Let $g$ be a special Bernstein function (e.g., a complete Bernstein function) with associated rate function $r=r[g]$ such that

$$
\operatorname{tr}(t) \rightarrow \infty \text { as } t \rightarrow \infty \text {. }
$$

Let $-A$ be the generator of a bounded $C_{0}$-semigroup $(T(s))_{s \geq 0}$ such that $z=0$ is an accumulation point of $\sigma(A)$. Then, whenever $\epsilon:(0, \infty) \rightarrow(0, \infty)$ is a decreasing function with $\lim _{t \rightarrow \infty} \epsilon(t)=0$, there exists $y \in \operatorname{ran}(g(A))$ such that

$$
\sup _{t \geq 1} \frac{\left\|\mathrm{C}_{t}(A) y\right\|}{\epsilon(t) r(t)}=\infty \text {. }
$$

Proof. By hypothesis we find $0 \neq z_{n}=\left|z_{n}\right| e^{i \theta_{n}} \in \sigma(A), n \in \mathbb{N}$, with $\theta_{n} \in$ $[-\pi / 2, \pi / 2]$ and $z_{n} \rightarrow 0$ as $n \rightarrow \infty$. Then

$$
t_{n}:=1 /\left|z_{n}\right| \rightarrow \infty \quad(n \rightarrow \infty) .
$$

Since $\operatorname{tr}(t) \rightarrow \infty, t \rightarrow \infty$, we may replace $\epsilon(t)$ by $\max \left(\epsilon(t),[\operatorname{tr}(t)]^{-1}\right), t \geq 1$ and suppose without loss of generality that

$$
\beta:=\inf _{n \in \mathbb{N}} \epsilon\left(t_{n}\right) t_{n} r\left(t_{n}\right)>0 .
$$

Furthermore, $\delta:=\inf _{n \in \mathbb{N}}\left|1-e^{-e^{i \theta_{n}}}\right|>0$, since the function $\theta \mapsto\left|1-e^{-e^{i \theta}}\right|$ is continuous and does not have a zero in $[-\pi / 2, \pi / 2]$.

By the spectral inclusion Theorem 2.2, Theorem 2.8 and Proposition 4.3 we obtain

$$
\begin{aligned}
\left\|g(A) \mathrm{C}_{t_{n}}(A)\right\| & =\left\|\left(\mathrm{C}_{t_{n}} \cdot g\right)(A)\right\| \geq \sup _{\lambda \in \sigma(A)}\left|\left(\mathrm{C}_{t_{n}} \cdot g\right)(\lambda)\right| \geq\left|\mathrm{C}_{t_{n}}\left(z_{n}\right) g\left(z_{n}\right)\right| \\
& =\frac{\left|1-e^{-t_{n} z_{n}}\right|}{t_{n}\left|z_{n}\right|}\left|g\left(z_{n}\right)\right|=\left|1-e^{-e^{i \theta_{n}}}\right| \cdot\left|g\left(z_{n}\right)\right| \geq \frac{\delta}{3 e^{2}} r\left(t_{n}\right)
\end{aligned}
$$

for each $n \in \mathbb{N}$. Consequently, since $\epsilon\left(t_{n}\right) \rightarrow 0$ as $n \rightarrow \infty$, one has

$$
\sup _{n \in \mathbb{N}} \frac{\left\|g(A) \mathrm{C}_{t_{n}}(A)\right\|}{\epsilon\left(t_{n}\right) r\left(t_{n}\right)}=\infty .
$$

Each operator $g(A) \mathrm{C}_{t_{n}}(A)$ is similar to its restriction to $\operatorname{dom}(A)$ by means of the isomorphism $(I+A)^{-1}: X \rightarrow \operatorname{dom}(A)$. By the uniform boundedness principle there is $x \in \operatorname{dom}(A) \subseteq \operatorname{dom}(g(A))$ such that

$$
\sup _{n \in \mathbb{N}} \frac{\left\|g(A) \mathrm{C}_{t_{n}}(A) x\right\|_{\operatorname{dom}(A)}}{\epsilon\left(t_{n}\right) r\left(t_{n}\right)}=\infty .
$$

On the other hand, setting $y:=g(A) x$ we obtain

$$
\begin{aligned}
\left\|g(A) \mathrm{C}_{t_{n}}(A) x\right\|_{\operatorname{dom}(A)} & =\left\|g(A) \mathrm{C}_{t_{n}}(A) x\right\|+\left\|A \mathrm{C}_{t_{n}}(A) g(A) x\right\| \\
& \leq\left\|\mathrm{C}_{t_{n}}(A) y\right\|+\frac{M+1}{t_{n}}\|g(A) x\| \\
& \leq\left\|\mathrm{C}_{t_{n}}(A) y\right\|+\frac{(M+1) \epsilon\left(t_{n}\right) r\left(t_{n}\right)}{\beta}\|g(A) x\|,
\end{aligned}
$$


where as always $M:=\sup _{s \geq 0}\|T(s)\|$. It follows that

$$
\sup _{n \in \mathbb{N}} \frac{\left\|\mathrm{C}_{t_{n}}(A) y\right\|}{\epsilon\left(t_{n}\right) r\left(t_{n}\right)}=\infty
$$

and this concludes the proof.

Remark 4.7. Clearly, (4.1) can be rewritten as

$$
\sup _{t \geq 1} \frac{\left\|\mathrm{C}_{t}(A) y\right\|}{\epsilon(t) g(1 / t)}=\infty \text {. }
$$

Remark 4.8. For general Bernstein functions $g$ with rate $r=r[g]$ satisfying $\operatorname{tr}(t) \rightarrow$ $\infty$ as $t \rightarrow \infty$ the conclusion of Theorem 4.6 remains true if one requires the stronger spectral condition that $z=0$ is an accumulation point of

$$
\sigma(A) \cap\left\{z \in \mathbb{C}_{+}|| \arg (z) \mid \leq \theta\right\}
$$

for some angle $\theta \in[0, \pi / 2)$. (The proof is similar to that of Theorem 4.6, but employs Proposition 4.2 instead of Proposition 4.3) This applies in particular to semigroups of non-invertible isometries, since then the whole halfplane $\overline{\mathbb{C}}_{+}$is contained in $\sigma(A)$. A fortiori, it applies also when one has a closed invariant subspace where the semigroup is like that.

\section{EXAMPLES}

We now discuss several examples important for applications. Let in this section $-A$ be the generator of a bounded $C_{0}$-semigroup $(T(s))_{s \geq 0}$ on a Banach space $X$. For a general theory of fractional powers and logarithms of $A$ we refer to 23 , Chapter 3].

Fractional Powers and Polynomial Rates. For $0<\alpha<1$ the function $g(z):=$ $z^{\alpha}$ is a complete Bernstein function with representation

$$
z^{\alpha}=\int_{0}^{\infty}\left(1-e^{-z s}\right) \mu(\mathrm{d} s), \quad \mu(\mathrm{d} s)=\frac{\alpha}{\Gamma(1-\alpha)} s^{-(\alpha+1)} \mathrm{d} s,
$$

where $\Gamma(\cdot)$ is the Gamma function, see [41, p. 219]. The operator $g(A)=\left(z^{\alpha}\right)(A)$ equals the commonly used fractional power $A^{\alpha}$ of $A$, see [23, Chapter 3]. The associated potential is

$$
z^{-\alpha}=\frac{1}{\Gamma(\alpha)} \int_{0}^{\infty} t^{\alpha-1} e^{-t z} \mathrm{~d} t .
$$

By Proposition 4.2, the associated rate satisfies $r(t) \sim(1 / t)^{\alpha}=t^{-\alpha}\left(\right.$ take $\left.z_{t}=1 / t\right)$. Theorems 3.4 and 4.6 then yield the following result.

Theorem 5.1. Let $-A$ be the generator of a bounded $C_{0}$-semigroup on a Banach space $X$ and let $\alpha \in(0,1)$.

a) For each $x \in \operatorname{ran}\left(A^{\alpha}\right)$

$$
\left\|\mathrm{C}_{t}(A) x\right\|=\mathrm{O}\left(t^{-\alpha}\right) \quad \text { as } t \rightarrow \infty .
$$

b) If the semigroup is mean-ergodic, then for each $x \in \operatorname{ran}\left(A^{\alpha}\right)$

$$
\left\|\mathrm{C}_{t}(A) x\right\|=\mathrm{o}\left(t^{-\alpha}\right) \quad \text { as } t \rightarrow \infty .
$$


c) If $z=0$ is an accumulation point of $\sigma(A)$, then for any decreasing function $\epsilon:(0, \infty) \rightarrow(0, \infty)$ with $\lim _{t \rightarrow \infty} \epsilon(t)=0$ there exists $y \in \operatorname{ran}\left(A^{\alpha}\right)$ such that

$$
\sup _{t \geq 1} \frac{t^{\alpha}\left\|\mathrm{C}_{t}(A) y\right\|}{\epsilon(t)}=\infty
$$

If $\operatorname{ker}(A)=\{0\}$, then one can clearly formulate condition $x \in \operatorname{ran}\left(A^{\alpha}\right)$ in the above result as $x \in \operatorname{dom}\left(A^{-\alpha}\right)$. Thus, the result can be given a form similar to Theorem 5.4 on logarithmic rates below.

Logarithmic Rates. To find a Bernstein function $g$ with an associated rate $r(t) \sim$ $1 / \log t$ as $t \rightarrow \infty$ we consider the function

$$
g(z)=\frac{z-1}{\log z}, \quad z>0 .
$$

By Example 2.9, $f=1 / g$ is a Stieltjes function, whence by Theorem 2.10 $g$ is a complete Bernstein function $g \sim(0,0, \mu)$. However, a closed expression for $\mu$ seems to be unknown, cf. [41, p. 236-237]. By inserting $z_{t}=1 / t$ we obtain $r(t) \sim \frac{1-1 / t}{\log t}$ for the corresponding rate function (Proposition 4.2). Hence, by Theorem 3.4 we have

$$
\left\|\mathrm{C}_{t}(A) x\right\|=\mathrm{O}(1 / \log t) \quad \text { as } t \rightarrow \infty \text { for each } x \in \operatorname{ran}(g(A))
$$

and $1 / \log t$ is optimal on $\operatorname{ran}(g(A))$ under the spectral conditions of Theorem 4.6.

The Operator Logarithm. Suppose that $A$ is injective. Then Theorem 5.1 tells that if $0<\alpha<1$ one has the rate $\mathrm{O}\left(t^{-\alpha}\right)$ for $\mathrm{C}_{t}(A) x$ and $x \in \operatorname{dom}\left(A^{-\alpha}\right)$, and this rate is optimal in the sense of part (iii) of Theorem [5.1. We claim that the analogous result holds with a $\operatorname{logarithmic}$ rate for $x \in \operatorname{dom}(\log A)$.

To begin with, let us say a few words on the operator $\log$ arithm. Since $\log z /(z-$ 1 ) is Stieltjes (Example 2.9), the function $(z \log z) /(z-1)$ is a complete Bernstein function (Theorem 2.10). But then by Lemma 2.5

$$
\left(\frac{z \log z}{z-1}\right) \frac{1}{1+z} \in \mathrm{A}_{+}^{1}\left(\mathbb{C}_{+}\right) \text {. }
$$

Since

we have

$$
\frac{z-1}{z+1}=1-\frac{2}{1+z} \in \mathrm{A}_{+}^{1}\left(\mathbb{C}_{+}\right)
$$

Moreover

$$
\frac{z}{(1+z)^{2}} \log z=\left(\frac{z-1}{z+1}\right)\left(\frac{z \log z}{z-1}\right) \frac{1}{1+z} \in \mathrm{A}_{+}^{1}\left(\mathbb{C}_{+}\right) \text {. }
$$

$$
\frac{z}{(1+z)^{2}}=\frac{1}{1+z}-\frac{1}{(1+z)^{2}} \in \mathrm{A}_{+}^{1}\left(\mathbb{C}_{+}\right) \text {. }
$$

Hence, if $A$ is injective, then $z(1+z)^{-2}$ is a regularizer for $\log z$, and therefore $\log A$ is defined in the extended HP-calculus for $A$.

One can approach the operator $\log A$ also via resolvents. Namely, for any fixed $\lambda \in \mathbb{C}$ with $|\operatorname{Im} \lambda|>\pi$ we have the following two representations of the function $(\lambda-\log z)^{-1}$ for $z \in \mathbb{C}_{+}$:

$$
\frac{1}{\lambda-\log z}=\int_{0}^{\infty} \frac{-1}{(\lambda-\log t)^{2}+\pi^{2}} \frac{\mathrm{d} t}{t+z}=\int_{0}^{\infty}\left[\int_{0}^{\infty} \frac{-e^{-t s} \mathrm{~d} t}{(\lambda-\log t)^{2}+\pi^{2}}\right] e^{-s z} \mathrm{~d} s .
$$

The first is the classical (Stieltjes type) representation used by Nollau [36] to define $\log A$ for sectorial operators $A$. It is proved by a standard contour deformation 
argument, cf. [23, Lemma 3.5.1]. The second (Laplace type) representation follows easily from the first. It is important for us since

$$
\int_{0}^{\infty} \int_{0}^{\infty}\left|\frac{-e^{-t s}}{(\lambda-\log t)^{2}+\pi^{2}}\right| \mathrm{d} t \mathrm{~d} s=\int_{0}^{\infty} \frac{1}{\left|(\lambda-\log t)^{2}+\pi^{2}\right|} \frac{\mathrm{d} t}{t}<\infty .
$$

This shows that $(\lambda-\log z)^{-1} \in \mathrm{A}_{+}^{1}\left(\mathbb{C}_{+}\right)$. From abstract functional calculus theory [23. Cor. 1.2.4] it follows that

$$
\left(\frac{1}{\lambda-\log z}\right)(A)=(\lambda-\log A)^{-1}
$$

and that our definition of $\log A$ yields the same operator as the sectorial functional calculus [23, Remark 3.3.3].

Let us now turn to the problem of rates for $\mathrm{C}_{t}(A) x$ when $x \in \operatorname{dom}(\log A)$. Since $(z-1) / \log z$ is a Bernstein function, the following abstract result is useful.

Theorem 5.2. Let $-A$ be the generator of a bounded $C_{0}$-semigroup $(T(s))_{s \geq 0}$ on a Banach space $X$ with $M:=\sup _{s \geq 0}\|T(s)\|$. Furthermore, let $f$ be a function such that $f(A)$ is defined in the extended HP-calculus and, for some $0 \neq \lambda \in \mathbb{C}$,

$$
g(z):=\frac{z-\lambda}{f(z)} \quad \text { is a Bernstein function. }
$$

Then the following statements hold:

a) For each $x \in \operatorname{dom}(f(A))$

$$
\left\|\mathrm{C}_{t}(A) x\right\| \leq \frac{c M}{|f(1 / t)|}(\|x\|+\|f(A) x\|) \quad(t \geq 1)
$$

where the constant $c$ depends only on $f$ and $\lambda$.

b) If the semigroup is mean ergodic and $t /|f(1 / t)| \rightarrow \infty$ as $t \rightarrow \infty$, then

$$
\left\|\mathrm{C}_{t}(A) x\right\|=\mathrm{o}\left(\frac{1}{|f(1 / t)|}\right) \quad \text { for each } x \in \operatorname{dom}(f(A)) \text {. }
$$

Proof. We write $r=r[g]$. From the definition of $g$ we obtain $\lambda=z-g(z) f(z)$. Multiplying with $\mathrm{C}_{t}(z)$ yields

$$
\lambda \mathrm{C}_{t}(z)=\frac{t z \mathrm{C}_{t}(z)}{t}-\left(g(z) \mathrm{C}_{t}(z)\right) f(z) \quad(t>0)
$$

and inserting $A$ yields

$$
|\lambda|\left\|\mathrm{C}_{t}(A) x\right\| \leq \frac{M+1}{t}\|x\|+2 M r(t)\|f(A) x\| \quad(t>0, x \in \operatorname{dom}(f(A))) .
$$

Since $r(t) \geq r(1) / t$ for $t \geq 1$ by Theorem 3.2 we arrive at

$$
\left\|\mathrm{C}_{t}(A) x\right\| \leq r(t) \frac{2 M}{|\lambda|}\left(\frac{1}{r(1)}+1\right)(\|x\|+\|f(A) x\|) \quad(t \geq 1) .
$$

By Proposition 4.2 and its proof we have

$$
r(t) \leq e|g(1 / t)|=e \frac{(1 / t)-\lambda}{|f(1 / t)|} \leq \frac{e(1+|\lambda|)}{|f(1 / t)|}
$$

Combining this with the previous we obtain

$$
\left\|\mathrm{C}_{t}(A) x\right\| \leq \frac{1}{|f(1 / t)|} \frac{4 M e}{|\lambda|}\left(\frac{1}{r(1)}+1\right)(\|x\|+\|f(A) x\|) \quad(t \geq 1)
$$


for $x \in \operatorname{dom}(f(A))$, proving a). Then b) follows from the above computations by an argument similar to that from the proof of Theorem 3.4 .

Remark 5.3. Let $g \neq 0$ be a complete Bernstein function with $\lim _{t \nearrow_{\infty}} g(t) / t=0$. Then by Theorem 2.12 it follows that $f(z):=g(z)-g(1 / z)$ is such that $f(z) /(z-1)$ is Stieltjes. As in the case of the logarithm, it follows from Theorem 2.10 that $(z-1) / f(z)$ and $q(z):=z f(z) /(z-1)$ are complete Bernstein functions. Hence

$$
\frac{z}{(1+z)^{2}} f(z)=\left(\frac{z-1}{z+1}\right) q(z) \frac{1}{1+z} \in \mathrm{A}_{+}^{1}\left(\mathbb{C}_{+}\right),
$$

and as above it follows that if $A$ is injective, then $f(A)$ is defined in the extended HP-calculus for $A$. Hence, for injective $A$, Theorem 2.12 provides a large class of functions satisfying the conditions of Theorem 5.4.

We are now in a position to state and prove our final result.

Theorem 5.4. Let $-A$ be the generator of a bounded $C_{0}$-semigroup on a Banach space $X$, and suppose that $A$ is injective.

a) For each $x \in \operatorname{dom}(\log A)$

$$
\left\|\mathrm{C}_{t}(A) x\right\|=\mathrm{O}\left(\frac{1}{\log t}\right) \quad \text { as } t \rightarrow \infty \text {. }
$$

b) If $\operatorname{ran}(A)$ is dense in $X$ then for each $x \in \operatorname{dom}(\log A)$

$$
\left\|\mathrm{C}_{t}(A) x\right\|=\mathrm{o}\left(\frac{1}{\log t}\right) \quad \text { as } t \rightarrow \infty .
$$

c) If $z=0$ is an accumulation point of $\sigma(A)$, then for any decreasing function $\epsilon:(0, \infty) \rightarrow(0, \infty)$ with $\lim _{t \rightarrow \infty} \epsilon(t)=0$ there exists $y \in \operatorname{dom}(\log A)$ such that

$$
\sup _{t \geq 1} \frac{\log t\left\|\mathrm{C}_{t}(A) y\right\|}{\epsilon(t)}=\infty
$$

Proof. As pointed out above, $(z-1) / \log z$ is a Bernstein function, so the statements a) and b) follow from Theorem 5.2 The argument for c) follows closely the proof of Theorem 4.6. We fix $\tau>\pi$ and $\lambda:=i \tau$. Suppose that $0 \neq z=|z| e^{i \theta} \in \sigma(A)$ such that $|z| \leq e^{-(\tau+\pi / 2)}$. Then $|\theta| \leq \pi / 2$ and

$$
|\lambda-\log z|^{2}=(\log |z|)^{2}+(\tau-\theta)^{2} \leq(\log |z|)^{2}+\left(\tau+\frac{\pi}{2}\right)^{2} \leq 2(\log |z|)^{2} .
$$

Since $(\lambda-\log z)^{-1} \in \mathrm{A}_{+}^{1}\left(\mathbb{C}_{+}\right)$we can apply the spectral inclusion Theorem 2.2 and infer - with $t:=1 /|z|$ - that

$$
\left\|\mathrm{C}_{t}(A)(\lambda-\log A)^{-1}\right\| \geq\left|\frac{\mathrm{C}_{t}(z)}{\lambda-\log z}\right| \geq \frac{\delta}{\sqrt{2}|\log | z||},
$$

where $\delta:=\inf _{|\varphi| \leq \pi / 2}\left|1-e^{-e^{i \varphi}}\right|>0$ as in the proof of Theorem 4.6

Now, by assumption there is a sequence $0 \neq z_{n} \in \sigma(A)$ with $z_{n} \rightarrow 0$. Without loss of generality we may suppose that $\left|z_{n}\right| \leq e^{-\tau-\pi / 2}$ for all $n \in \mathbb{N}$. Hence, with $t_{n}:=1 /\left|z_{n}\right| \rightarrow \infty$

$$
\left\|\mathrm{C}_{t_{n}}(A)(\lambda-\log A)^{-1}\right\| \geq \frac{\delta}{\sqrt{2} \log t_{n}} \quad(n \in \mathbb{N}) .
$$


The uniform boundedness principle yields $x \in X$ such that for $y:=(\lambda-\log A)^{-1} x \in$ $\operatorname{dom}(\log A)$ we have

$$
\sup _{n \in \mathbb{N}} \frac{\log \left(t_{n}\right)\left\|C_{t_{n}}(A) x\right\|}{\epsilon\left(t_{n}\right)}=\infty
$$

and this concludes the proof.

\section{Appendix A.}

Theorem A.1. Let $-A$ be the generator of a bounded $C_{0}$-semigroup $(T(s))_{s \geq 0}$ on a Banach space $X$. Suppose $\varphi:(0, \infty) \rightarrow(0, \infty)$ is a function such that $\varphi(t) \searrow 0$ as $t \rightarrow \infty$, and

$$
\left\|\mathrm{C}_{t}(A) x\right\|=\mathrm{O}(\varphi(t)) \quad \text { for every } x \in \operatorname{dom}_{\infty}(A):=\cap_{n=0}^{\infty} \operatorname{dom}\left(A^{n}\right) .
$$

Then $A$ is invertible.

Proof. By assumption it follows that $\operatorname{dom}_{\infty}(A) \subset \overline{\operatorname{ran}}(A)$. Since $-A$ is the generator of a $C_{0}$-semigroup, $\operatorname{dom}_{\infty}(A)$ is dense in $X$, so that $\overline{\operatorname{ran}}(A)=X$. Thus since $\operatorname{ker}(A)=\{0\}$, it suffices to prove that $\operatorname{ran}(A)$ is closed. By hypothesis, for $x \in$ $\operatorname{dom}_{\infty}(A)$ there is $c(x)$ such that

$$
\left\|\mathrm{C}_{t}(A) x\right\| \leq c(x) \varphi(t) \quad(t \geq 1) .
$$

For $n \in \mathbb{N}$ we consider $\operatorname{dom}\left(A^{n}\right)$ as a Banach space with the graph norm

$$
\|x\|_{\operatorname{dom}\left(A^{n}\right)}:=\sum_{j=0}^{n}\left\|A^{j} x\right\| \quad\left(x \in \operatorname{dom}\left(A^{n}\right)\right) .
$$

The space $\operatorname{dom}_{\infty}(A)$ is a Fréchet space with respect to the increasing sequence of norms $\left(\|\cdot\|_{\operatorname{dom}\left(A^{n}\right)}\right)_{n \geq 0}$. By (A.1) and the principle of uniform boundedness for Fréchet spaces [38, Theorem 2.6], we obtain that there exist $n \in \mathbb{N} \cup\{0\}$ and $c>0$ such that

$$
\left\|\mathrm{C}_{t}(A) x\right\| \leq c \varphi(t)\|x\|_{\operatorname{dom}\left(A^{n}\right)} \quad \text { for all } \quad t \geq 1, x \in \operatorname{dom}_{\infty}(A) .
$$

Since $\operatorname{dom}_{\infty}(A)$ is a core for the closed operator $A^{n}$, we then have

$$
\left\|\mathrm{C}_{t}(A) x\right\| \leq c \varphi(t)\|x\|_{\operatorname{dom}\left(A^{n}\right)} \quad \text { for all } \quad t \geq 1, x \in \operatorname{dom}\left(A^{n}\right) .
$$

Now $\mathrm{C}_{t}(A) X \subseteq \operatorname{dom}(A)$ and

$$
t A \mathrm{C}_{t}(A)=A \int_{0}^{t} T(s) \mathrm{d} s=I-T(t) \text { for all } t>0 .
$$

Hence we have for every $1 \leq j \leq n$

$$
\left\|A^{j} \mathrm{C}_{t} x\right\| \leq \frac{\|I-T(t)\|}{t}\left\|A^{j-1} x\right\| \leq \frac{M+1}{t}\left\|A^{j-1} x\right\| \quad\left(x \in \operatorname{dom}\left(A^{n}\right), t>0\right),
$$

where $M:=\sup _{s \geq 0}\|T(s)\|$. From (A.2) it then follows that

$$
\left\|\mathrm{C}_{t}(A)\right\|_{\operatorname{dom}\left(A^{n}\right) \rightarrow \operatorname{dom}\left(A^{n}\right)} \leq c \varphi(t)+\frac{M+1}{t},
$$

where the right-hand side tends to zero as $t \rightarrow \infty$. Therefore, for $t \geq 1$ large enough the operator

$$
I-\mathrm{C}_{t}(A): \operatorname{dom}\left(A^{n}\right) \longrightarrow \operatorname{dom}\left(A^{n}\right)
$$

is invertible. This operator is similar to the operator

$$
I-\mathrm{C}_{t}(A): X \longrightarrow X
$$


by virtue of the isomorphism $(I+A)^{-n}: X \rightarrow \operatorname{dom}\left(A^{n}\right)$. Multiplying with $t$ yields that $S:=\int_{0}^{t}(I-T(s)) \mathrm{d} s$ is invertible on $X$. But for $x \in \operatorname{dom}(A)$

$$
S x=\int_{0}^{t}(I-T(s)) x \mathrm{~d} s=\int_{0}^{t} \int_{0}^{s} T(r) A x \mathrm{~d} r \mathrm{~d} s=\int_{0}^{t}(t-r) T(r) A x \mathrm{~d} r,
$$

and hence

$$
\|x\| \leq\left\|S^{-1}\right\|\left\|\int_{0}^{t}(I-T(s)) x \mathrm{~d} s\right\| \leq\left(C M t^{2} / 2\right)\|A x\|
$$

for large enough $t$ and for all $x \in \operatorname{dom}(A)$. This concludes the proof.

\section{Appendix B.}

In this Appendix we characterize those rate functions $r$ that appear as $r=[g]$ for some complete Bernstein function. We need the following lemma.

Lemma B.1. Let $g$ be a Bernstein function and let $r=r[g]$ the associated rate as in (3.2). Then, with $\tilde{r}(t)=\operatorname{tr}(t)$,

$$
(\mathcal{L} \tilde{r})(z)=\frac{g(z)-\frac{g(0+)}{2}}{z^{2}} \quad(\operatorname{Re} z>0) .
$$

In other words, $\operatorname{tr}(t)$ is the inverse Laplace transform of $[g(z)-g(0+) / 2] / z^{2}$.

Proof. Suppose first that $g(0+)=0$, so that $g(\lambda)=b \lambda+\int_{0+}^{\infty}\left(1-e^{-\lambda s}\right) \mu(\mathrm{d} s)$. Then

$$
\begin{aligned}
\int_{0}^{\infty} & t r(t) e^{-\lambda t} \mathrm{~d} t=\int_{0}^{\infty} e^{-\lambda t}\left[b+\int_{0+}^{t} s \mu(\mathrm{d} s) \mathrm{d} t+t \int_{t+}^{\infty} \mu(\mathrm{d} s)\right] \mathrm{d} t \\
= & \frac{b}{\lambda}+\int_{0+}^{\infty}\left(\int_{s}^{\infty} e^{-\lambda t} \mathrm{~d} t\right) s \mu(\mathrm{d} s)+\int_{0+}^{\infty}\left(\int_{0}^{s} t e^{-\lambda t} \mathrm{~d} t\right) \mu(\mathrm{d} s) \\
& =\frac{b}{\lambda}+\frac{1}{\lambda} \int_{0+}^{\infty} s e^{-\lambda s} \mu(\mathrm{d} s)+\int_{0+}^{\infty}\left(-\frac{s e^{-\lambda s}}{\lambda}+\frac{1-e^{-\lambda s}}{\lambda^{2}}\right) \mu(\mathrm{d} s) \\
& =\frac{b}{\lambda}+\frac{1}{\lambda^{2}} \int_{0+}^{\infty}\left(1-e^{-\lambda s}\right) \mu(\mathrm{d} s)=\frac{g(\lambda)}{\lambda^{2}}
\end{aligned}
$$

for all $\lambda>0$. In the general case we have to add $\frac{a}{2} \int_{0}^{\infty} t e^{-t \lambda} \mathrm{d} t=\frac{g(0+)}{2 \lambda^{2}}$ in each step of the computation.

Corollary B.2. If $r=r[g]$ for a Bernstein function $g$ such that

$$
g(0+)=\lim _{t \rightarrow \infty} r(t)=0,
$$

then $\operatorname{tr}(t)$ is the inverse Laplace transform of $g(z) / z^{2}$.

Now we can state the main result of this Appendix.

Theorem B.3. A function $r:(0, \infty) \rightarrow(0, \infty)$ is of the form $r=r[g]$ for some complete Bernstein function $g$ if, and only if, $t \mapsto \operatorname{tr}(t)$ is a Bernstein function.

Proof. Suppose that $r=r[g]$ for some complete Bernstein function $g$. Then $g-$ $g(0+) / 2$ is a complete Bernstein function, too, whence by [41, Theorem 6.2] there exists a Bernstein function $h$ such that

$$
g(\lambda)-\frac{g(0+)}{2}=\lambda^{2}(\mathcal{L} h)(\lambda) \quad(\lambda>0) .
$$


Lemma B.1 together with the uniqueness of the Laplace transform implies that $h(t)=\operatorname{tr}(t)$ for $t>0$, and hence $t \mapsto \operatorname{tr}(t)$ is a Bernstein function.

Conversely, suppose that $h(t)=\operatorname{tr}(t)$ is a Bernstein function. Then $a:=$ $2 \lim _{t \rightarrow \infty} h(t) / t$ exists and $h$ is Laplace transformable. By [41, Theorem 6.2] again,

$$
g(\lambda):=\frac{a}{2}+\lambda^{2}(\mathcal{L} h)(\lambda) \quad(\lambda>0)
$$

is a complete Bernstein function. A short computation based on the Bernstein representation of $h$ yields that

$$
\lim _{\lambda \searrow 0} \lambda^{2}(\mathcal{L} h)(\lambda)=\lim _{t \rightarrow \infty} \frac{h(t)}{t}=\frac{a}{2},
$$

cf. the proof of 41, Theorem 6.2]. It follows that $a=g(0+)$ and, by LemmaB.1 and the uniqueness of the Laplace transform, $r[g](t)=h(t) / t=r(t)$ for all $t>0$.

\section{Acknowledgements}

The authors are grateful to the referee for his/her very careful reading of the manuscript and useful remarks and suggestions.

\section{REFERENCES}

[1] I. Assani and M. Lin, On the one-sided ergodic Hilbert transform, Ergodic theory and related fields, Contemporary Math., 430, Providence, RI, 2007, 21-39.

[2] A. V. Balakrishnan, An operational calculus for infinitesimal generators of semigroups, Trans. Amer. Math. Soc. 91 (1959), 330-353.

[3] F. E. Browder, On the iteration of transformations in noncompact minimal dynamical systems, Proc. Amer. Math. Soc. 9 (1958), 773-780.

[4] P. Butzer and A. Gessinger, Ergodic theorems for semigroups and cosine operator functions at zero and infinity with rates; applications to partial differential equations. A survey, Mathematical analysis, wavelets, and signal processing, Contemporary Math. 190 (1995), $67-94$.

[5] P. Butzer and U. Westphal, The mean ergodic theorem and saturation, Indiana Univ. Math. J. 20 (1971), 1163-1174.

[6] P. Clément and J. Prüss, Completely positive measures and Feller semigroups, Math. Ann. 287 (1990), 73-105.

[7] G. Cohen, C. Cuny and M. Lin, The one-sided ergodic Hilbert transform in Banach spaces, Studia Mathematica 196 (2010), 251-263.

[8] G. Cohen, C. Cuny and M. Lin, On convergence of power series of $L_{p}$ contractions, Banach Center Publications, 2011, to appear.

[9] G. Cohen and M. Lin, Laws of large numbers with rates and the one-sided ergodic Hilbert transform, Illinois J. Math. 47 (2003), 997-1031.

[10] G. Cohen and M. Lin, Extensions of the Menchoff-Rademacher theorem with applications to ergodic theory, Israel J. Math. 148 (2005), 41-86.

[11] G. Cohen and M. Lin, The one-sided ergodic Hilbert transform of normal contractions, in: Characteristic functions, scattering functions and transfer functions - the Moshe Livsic memorial volume. Birkhäuser, Basel, 2009, 77-98.

[12] C. Cuny, Pointwise ergodic theorems with rate and application to limit theorems for stationary processes, Stochastics and Dynamics, 11 (2011), 135-155.

[13] C. Cuny, On the a.s. convergence of the one-sided ergodic Hilbert transform, Ergodic Th. Dynamical Sys. 29 (2009), 1781-1788.

[14] C. Cuny and M. Lin, Pointwise ergodic theorems with rate and application to the CLT for Markov chains, Ann. Inst. H. Poincare, ser. Prob. and Stat. 45 (3) (2009), 710-733.

[15] R. deLaubenfels, Automatic extensions of functional calculi, Studia Math. 114 (1995), 237259 . 
[16] Y. Derriennic, Some aspects of recent works on limit theorems in ergodic theory with special emphasis on the "central limit theorem", Discrete Contin. Dyn. Syst. (Ser. A) 15 (2006), $143-158$.

[17] Y. Derriennic and M. Lin, Fractional Poisson equations and ergodic theorems for fractional coboundaries, Israel J. Math. 123 (2001), 93-130.

[18] N. Dunford, Spectral theory. I. Convergence to projections, Trans. Amer. Math. Soc. 54 (1943), 185-217.

[19] K.-J. Engel and R. Nagel, One-Parameter Semigroups for Linear Evolution Equations, Graduate Texts in Mathematics 194, Berlin, Springer, 2000.

[20] V. F. Gaposhkin, On the rate of decrease of the probabilities of $\epsilon$-deviations for means of stationary processes, Mat. Zametki 64 (1998), 366-372 (in Russian); translation in Math. Notes 64 (1998), 316-321.

[21] J. Goldstein, C. Radin, and R. Showalter, Convergence rates of ergodic limits for semigroups and cosine functions, Semigroup Forum 16 (1978), 89-95.

[22] A. Gomilko, M. Haase, and Yu. Tomilov, On rates in mean ergodic theorems, Math. Res. Letters 18 (2011), 201-213.

[23] M. Haase, The Functional Calculus for Sectorial Operators. Operator Theory: Advances and Applications 169, Birkhäuser, Basel, 2006.

[24] M. Haase and Yu. Tomilov, Domain characterizations of certain functions of power-bounded operators, Studia Math. 196 (2010), 265-288.

[25] E. Hille abd R. S. Phillips, Functional Analysis and Semi-Groups, 3rd printing of rev. ed. of 1957, Colloq. Publ. 31, AMS, Providence, RI, 1974.

[26] F. Hirsch, Transformation de Stieltjes et fonctions opérant sur les potentiels abstraits, Théorie du potentiel et analyse harmonique (Journées Soc. Math. France, Inst. Recherche Math. Avancée, Strasbourg, 1973), Lecture Notes in Math. 404, Springer, Berlin, 1974, 149-163.

[27] F. Hirsch, Familles d'opérateurs potentiels, Ann. Inst. Fourier 254 (1975), 263-288.

[28] F. Hirsch, Domaines d'opérateurs représentés comme intégrales de résolvantes, J. Functional Analysis 23 (1976), 199-217.

[29] A. G. Kachurovskii, Rates of convergence in ergodic theorems, Uspekhi Mat. Nauk 51 (1996), 73-124 (in Russian); translation in Russian Math. Surveys 51 (1996), 653-703.

[30] A. G. Kachurovskii and A. V. Reshetenko, On the rate of convergence in von Neumann's ergodic theorem with continuous time, Mat. Sb. 201 (2010), 25-32 (in Russian); translation in Sb. Math. 201 (2010), 493-500.

[31] Th. Kaluza, Über die Koeffizienten reziproker Potenzreihen, Math. Z. 28 (1928), 161-170.

[32] U. Krengel, Ergodic Theorems. With a supplement by Antoine Brunel. De Gruyter Studies in Mathematics 6, Berlin, Walter de Gruyter, 1985.

[33] U. Krengel and M. Lin, On the range of the generator of a Markovian semigroup, Math. Z. 185 (1984), 553-565.

[34] M. Lin, On the uniform ergodic theorem, Proc. Amer. Math. Soc. 43 (1974), 337-340.

[35] M. Lin, On the uniform ergodic theorem. II, Proc. Amer. Math. Soc. 46 (1974), 217-225.

[36] V. Nollau, Über den Logarithmus abgeschlossener Operatoren in Banachschen Räumen, Acta Sci. Math. (Szeged) 30 (1969), 161-174.

[37] R. S. Phillips, On the generation of semigroups of linear operators, Pacific J. Math. 2 (1952), 343-369.

[38] W. Rudin, Functional Analysis, 2nd ed., International Series in Pure and Applied Mathematics, NY, McGraw-Hill, 1991.

[39] S.-Y. Shaw, Convergence rates of ergodic limits and associated solutions, J. Approximation Theory 75 (1993), 157-166.

[40] R. L. Schilling, Subordination in the sense of Bochner and a related functional calculus, J. Austral. Math. Soc. Ser. A 64 (1998), 368-396.

[41] R. Schilling, R. Song, and Z. Vondraček, Bernstein functions, de Gruyter Studies in Mathematics 37, Walter de Gruyter, Berlin, 2010.

[42] U. Westphal, A generalized version of the Abelian mean ergodic theorem with rates for semigroup operators and fractional powers of infinitesimal generators, Results in Math. 34 (1998), 381-394.

[43] D. V. Widder, The Laplace Transform, Princeton Mathematical Series 6, Princeton University Press, Princeton, 1941. 
Faculty of Mathematics and Computer Science, Nicolas Copernicus University, ChoPIN STR. 12/18, 87-100 Toruń, Poland

E-mail address: gomilko@mat.uni.torun.pl

Delft Institute of Applied Mathematics, Delft University of Technology, P.O. Box 5031, 2600 GA Delft, The Netherlands

E-mail address: m.h.a.haase@tudelft.nl

Institute of Mathematics, Polish Academy of Sciences, Śniadeckich Str. 8, 00-956 Warszawa, Poland, and Faculty of Mathematics and Computer Science, Nicolas Copernicus University, Chopin Str. 12/18, 87-100 Torun, Poland,

E-mail address: tomilov@mat.uni.torun.pl 